\title{
Efficient near-field to far-field transformations for quasinormal modes of optical cavities and plasmonic resonators
}

\author{
Juanjuan Ren, ${ }^{1, *}$ Sebastian Franke, ${ }^{2}$ Andreas Knorr, ${ }^{2}$ Marten Richter, ${ }^{2}$ and Stephen Hughes ${ }^{1}$ \\ ${ }^{1}$ Department of Physics, Engineering Physics, and Astronomy, \\ Queen's University, Kingston, Ontario KrL 3N6, Canada \\ ${ }^{2}$ Technische Universität Berlin, Institut für Theoretische Physik, \\ Nichtlineare Optik und Quantenelektronik, Hardenbergstraße 36, 10623 Berlin, Germany
}

(Dated: December 19, 2019)

\begin{abstract}
We describe an efficient near-field to far-field transformation for optical quasinormal modes, which are the dissipative modes of open cavities and plasmonic resonators with complex eigenfrequencies. As an application of the theory, we show how one can compute the reservoir modes (or regularized quasinormal modes) outside the resonator, which are essential to use in both classical and quantum optics. We subsequently demonstrate how to efficiently compute the quantum optical parameters necessary in the theory of quantized quasinormal modes [Franke et al., Phys. Rev. Lett. 122, 213901 (2019)]. To confirm the accuracy of our technique, we directly compare with a Dyson equation approach currently used in the literature (in regimes where this is possible), and demonstrate several order of magnitude improvement for the calculation run times. We also introduce an efficient pole approximation for computing the quantized quasinormal mode parameters, since they require an integration over a range of frequencies. Using this approach, we show how to compute regularized quasinormal modes and quantum optical parameters for a full 3D metal dimer in under one minute on a standard desktop computer. Our technique is exemplified by studying the quasinormal modes of metal dimers and and a hybrid structure consisting of a gold dimer on top of a photonic crystal beam. In the latter example, we show how to compute the quantum optical parameters that describe a pronounced Fano resonance, using structural geometries that cannot practically be solved using a Dyson equation approach. All calculations for the spontaneous emission rates are confirmed with full-dipole calculations in Maxwell's equations and are shown to be in excellent agreement.
\end{abstract}

\section{INTRODUCTION}

Open-cavity photonic structure are widely used to enhance light-matter interaction at the nanoscale ${ }^{1,2}$, especially plasmonic cavities ${ }^{3-11}$, which allow one to enhance light-matter interactions without any fundamental bounds from diffraction - giving rise to high field electromagnetic hots spots. These optical hot spots have been used to strongly couple single molecules at room temperature and probe molecular optomechanics in the regime of surface enhanced Raman spectroscopy (SERS) ${ }^{12,13}$

In optical cavity physics, and especially cavity-QED (quantum electrodynamics), a few "mode" description for these cavities is of great benefit. A cavity mode description not only helps to explain the underlying physics of light-matter enhancement, but it can quantify the separation of radiative and nonradiative decay processes, and allow design insights into the important figures of merit. For closed systems without absorption, the cavity modes can be described by normal modes with real eigenfrequencies and infinite lifetimes ${ }^{14}$. This is often a good approximation for high $Q$ resonances (where $Q$ is the quality factor), but is still ambiguous in general ${ }^{15,16}$. However, most - if not all - systems are dissipative via radiation decay or/and absorption, and thus it is highly desirable to work with the correct dissipative modes.

One of the most powerful approaches to this problem is to use quasinormal modes $(\mathrm{QNMs})^{15-26}$, which are open cavity modes with complex eigenfrequency $\tilde{\omega}_{c}$, with a finite cavity lifetime $\tau_{c}=2 \pi /\left(-\operatorname{Im}\left(\tilde{\omega}_{c}\right)\right)$. The key ad- vantage of such a discrete modal approach is that often only a few QNMs are needed, and frequently just one QNM, which can be used to give an accurate description of light-matter interactions over a wide range of positions and frequencies ${ }^{15,16,21,22,25,27}$. In the semiclassical regime, one can rigorously compute a generalized effective mode volume ${ }^{15,19}$, the photon Green's function ${ }^{18,28}$, and the enhanced spontaneous emission rate (related to generalized Purcell factors $)^{28,29}$ in system-reservoir theory of quantum optics. Moreover, it has been recognized that quantization of QNMs would be a significant improvement in quantum optics/plasmonics theories ${ }^{30}$. Some progress has been made for one-dimensional dielectric structures ${ }^{31,32}$, but this approach does not lead to Fock states, typically used to expand multiphoton quantum field states. Recently, Franke et al. introduced a quantization for leaky optical cavities and plasmonic resonators based on $\mathrm{QNMs}^{33}$, which allows one to rigorously study multi-photon problems for open-cavity resonators, including dielectrics and metals. However, as input to the quantization theory, one requires the QNMs and reservoir modes ("regularized QNMs"28) outside the cavity region.

The QNMs represent a highly accurate description to the total field for positions inside the resonator but is not a good description for fields far outside, where one needs the continuous reservoir fields ${ }^{28}$. However, a special feature of the QNM field, $\tilde{\mathbf{f}}_{\mu}(\mathbf{r})$, is that they diverge exponentially outside the resonator, originating from the complex resonance frequency and the Silver-Müller radiation condition ${ }^{34}$. The locations for this divergent behav- 
ior depend mainly on the quality factor of optical cavity structure, and the shape of the mode, e.g., the spatial divergence may begin a few microns away from the resonator for typical low $Q$ plasmonic cavity $\operatorname{modes}^{35}$. At these locations, the QNMs are no longer a good representation of the fields outside the cavity, and generally one should only use the QNMs inside the cavity region; outside the cavity system, one needs the reservoir modes or regularized modes which become a function of continuous frequency. For example, one can obtain regularized fields outside the resonator from the solution inside by using a Dyson equation ${ }^{28}$,

$$
\tilde{\mathbf{F}}_{\mu}(\mathbf{R}, \omega) \approx \int_{V} d \mathbf{r G}^{\mathrm{B}}(\mathbf{R}, \mathbf{r}, \omega) \Delta \epsilon(\mathbf{r}, \omega) \tilde{\mathbf{f}}_{\mu}(\mathbf{r}),
$$

where $\mathbf{G}^{\mathrm{B}}(\mathbf{R}, \mathbf{r}, \omega)$ is the Green's function for the background medium and $\Delta \epsilon$ is the change in dielectric constant from the spatially-dependent resonator. In this way, one can obtain the fields everywhere, by only using the QNMs within the structure; other contributions can be included as needed, such as background contributions from evanescent modes if very near a metal surface ${ }^{28}$. Here we consider $\mathbf{R}$ to be outside the resonator, and $\mathbf{r}$ inside. We also note that the $\tilde{\mathbf{F}}_{\mu}(\mathbf{R}, \omega)$ are continuous in frequency (and these are expected to be accurate within the QNM spectral region of interest), while the $\tilde{\mathbf{f}}_{\mu}(\mathbf{r})$ is associated with the QNM complex frequency.

These regularized fields $\tilde{\mathbf{F}}_{\mu}$ have been shown to be highly accurate for obtaining the Purcell factor outside the resonator, and they properly converge in the far field ${ }^{28}$. Recently, it was also shown how these fields $\tilde{\mathbf{F}}_{\mu}$ are required for QNM quantization of arbitrary me$\mathrm{dia}^{33}$. While the Dyson approach works in principle, the computation can be tedious and impractical. For example, for the quantization scheme, one needs to integrate such fields from the outside region over a closed surface that surrounds the resonator, and over a wide range of frequencies; this approach requires significant computational memory and is extremely time consuming, especially for complex nanostructures. Indeed, even for simple metal dimer structures, computing $\tilde{\mathbf{F}}_{\mu}$ can take weeks on a high performance desktop computer, as we will also demontrate in this paper with several concrete examples. For more complex cavity structures, such as dimers on top of photonic crystal (PC) cavities $^{29}$, the general Dyson approach to obtain $\tilde{\mathbf{F}}_{\mu}$ is numerically intractable. Given the importance of using these frequency-continuous fields, $\tilde{\mathbf{F}}_{\mu}$, especially for connecting to observables, and for their use in quantized QNM theories, there is now an urgent need to develop more efficient and insightful way to obtain these QNM reservoir fields.

In this paper we present an efficient solution to this problem. We define a fictitious boundary surrounding the cavity that radiates to the far field through the appropriate surface currents flowing over the boundary; the sources inside a domain are replaced with sources on the surface of this domain, and the fields inside the domain can be chosen zero (field equivalence principle). We take advantage of this principle to introduce an efficient near field to far field (NF2FF) transformation for QNMs to obtain regularized fields that give the correct far field radiation flow. Moreover, the same transformation can be used to obtain QNMs in the far field, by either projecting in real frequency space (regularized QNM) or complex frequency space (divergent QNMs). For practical use in quantized QNM theories, we also show how the far fields can easily be computed via decomposing the near fields into spherical (3D) or cylindrical (2D) waves, which can then be propagated separately to the very far field regime ${ }^{36,37}$. The near fields we use as input are the QNMs fields at a surface close to the resonator. With these numerically computed QNMs, obtained for arbitrarily shaped 3D resonators, we demonstrate how one can then carry out NF2FF transformation with real frequencies and show how the results accurately converge.

The rest of our paper is organized as follows: In Sec. II, we introduce all the main theory needed in this paper. In II A, the core QNMs theory is presented, including the QNM Green's function expansion, classical Purcell factors and classical $\beta$ factors. Due to the divergent behaviour of the QNM fields, one current solutionthe Dyson equation approach - is introduced in IIB, which can be used to calculate the regularized (i.e., nondivergent) fields outside the resonator. This approach works, but is complicated and time consuming, especially for hybrid structures (i.e., a combined material created from dielectric and metal cavity parts). In II C, we introduce an alternative way to obtain these normalized fields using a NF2FF transformation. As further motivation to why we need these fields, the basic background of a recently developed quantized QNM theory ${ }^{33,38}$ is shown in Sec. IID, where we show how the regularized fields in addition to the QNMs are needed for the "quantum $S$ parameters"; these matrix elements relate to the commutation rules for quantization of the QNMs, and are only Kronecker delta symbols in the limit of no $\operatorname{loss}^{33,38}$. In II E, we introduce an efficient pole approximation, which simplifies the required integration over frequency for obtaining the $S$ factors, and we give analytical solutions for single and coupled QNM structures.

Using the above theory, various numerical examples are shown in Sec. III-IV. In III, we concentrate on single QNM results, and explore metal dimer gap modes, with different material losses, and investigate the resonance features, including the quality factor and complex eigenfrequency of the dominant localized plasmon mode; the field distribution are described in III A. In III B, the regularized field $\tilde{\mathbf{F}}(\mathbf{R}, \omega)$ are first obtained from Dyson approach, and compared with our newly developed NF2FF, where we show excellent agreement as the fields evolve to the far field. As an example application for classical optics, we show $\tilde{\mathbf{F}}(\mathbf{R}, \omega)$ at several far field surfaces, which are the fields that experiments can detect directly. Sections IIIC and IIID show detailed calculation for quantum parameters, including both nonradiative and radiative contributions, including detailed 
numerical convergence study. Section IIIE summarizes the computational run times to calculate the radiative contribution for both Dyson approach and NF2FF transformation, which is shown to reduce the calculation run times from days-weeks to under 1 minute using a standard workstation implemented in Matlab. In Sec. IV, we study a complex coupled QNM system, in a regime where the modes strongly overlap and the Purcell factors exceed 1 million. Specifically, we study a hybrid structure consisting of a gold ellipsoid dimer and a high-Q PC cavity, where two QNMs are overlapping in the frequency region of interest. The interfering modes yield a striking Fano-like resonance, which we show can be well explain using both the classical and quantum theory. In the latter case, the calculation using a Dyson to a NF2FF approach would require years of computational time, but are calculated here in minutes. The complex details of the Fano resonance feature are fully obtained using the quantized QNM approach in the bad cavity limit, without any fitting parameters, Finally, we present our conclusions in Sec. V.

\section{THEORY}

\section{A. Quasinormal modes, Green's function} expansions, classical Purcell factors and beta factors

The QNMs, $\tilde{\mathbf{f}}_{\mu}(\mathbf{r})$, are solutions to the Helmholtz equation,

$$
\nabla \times \nabla \times \tilde{\mathbf{f}}_{\mu}(\mathbf{r})-\left(\frac{\tilde{\omega}_{\mu}}{c}\right)^{2} \epsilon\left(\mathbf{r}, \tilde{\omega}_{\mu}\right) \tilde{\mathbf{f}}_{\mu}(\mathbf{r})=0
$$

subject to open boundary conditions, i.e., the SilverMüller radiation condition ${ }^{39}$. Here $\epsilon\left(\mathbf{r}, \tilde{\omega}_{\mu}\right)$ is the dielectric constant and $\tilde{\omega}_{\mu}=\omega_{\mu}-i \gamma_{\mu}$ the complex eigenfrequency with quality factor $Q_{\mu}=\omega_{\mu} / 2 \gamma_{\mu}$. Once normalized, the QNMs can be used to construct the transverse Green's function through ${ }^{18,28}$

$$
\mathbf{G}\left(\mathbf{r}, \mathbf{r}_{0}, \omega\right)=\sum_{\mu} A_{\mu}(\omega) \tilde{\mathbf{f}}_{\mu}(\mathbf{r}) \tilde{\mathbf{f}}_{\mu}\left(\mathbf{r}_{0}\right)
$$

for locations near (or within) the scattering geometry with volume $\mathrm{V}$, where the QNMs can form a complete basis $^{19,20}$. The photon Green's function, $\mathbf{G}\left(\mathbf{r}, \mathbf{r}_{0}, \omega\right)$, fulfills the equation:

$\nabla \times \nabla \times \mathbf{G}\left(\mathbf{r}, \mathbf{r}_{0}, \omega\right)-\frac{\omega^{2}}{c^{2}} \epsilon(\mathbf{r}, \omega) \mathbf{G}\left(\mathbf{r}, \mathbf{r}_{0}, \omega\right)=\frac{\omega^{2}}{c^{2}} \mathbf{1} \delta\left(\mathbf{r}-\mathbf{r}_{0}\right)$,

with corresponding radiation conditions, where $\mathbf{1}$ is a unit tensor and $c$ is light speed in vacuum.

Although there are several forms for $A_{\mu}(\omega)$, which are related by a sum relationship ${ }^{21,40}$, below we use

$$
A_{\mu}(\omega)=\frac{\omega}{2\left(\tilde{\omega}_{\mu}-\omega\right)}
$$

However, practically, when we limit the expansion to just a few modes, we use a slightly different form as an approximation:

$$
A_{\mu}(\omega) \approx \frac{\omega}{2\left(\tilde{\omega}_{\mu}-\omega\right)} \operatorname{Rect}\left(\frac{\omega-\omega_{\mu}-\omega_{\mu}^{\text {cut }}}{\omega-\omega_{\mu}}\right)
$$

where we now also include the top-hat or rectangular function: $\operatorname{Rect}(t)=1$, if $|t|<\frac{1}{2}$, else $\operatorname{Rect}(t)=0$. Later we show that a practical value for the cut-off is $\omega_{\mu}^{\text {cut }}=14 \gamma_{\mu}$ when we also compare with an efficient pole approximation, to evaluate the integrations over frequency.

We first consider a single QNM, $\mu=\mathrm{c}$, so the Green's function can be written as

$$
\mathbf{G}_{\mathrm{c}}\left(\mathbf{r}, \mathbf{r}_{0}, \omega\right) \approx A_{\mathrm{c}}(\omega) \tilde{\mathbf{f}}_{\mathrm{c}}(\mathbf{r}) \tilde{\mathbf{f}}_{\mathrm{c}}\left(\mathbf{r}_{0}\right)
$$

where again this holds only nearby the cavity region. This QNM expansion of Green's function can easily be used to compute the spontaneous emission (SE) rate and Purcell factor. For example, if one considers a quantum dipole emitter with dipole moment $\mathbf{d}\left(=\mathrm{d} \mathbf{n}_{\mathrm{d}}\right)$ at location $\mathbf{r}_{0}$, then the $\mathrm{SE}$ rate is 16

$$
\Gamma\left(\mathbf{r}_{0}, \omega\right)=\frac{2}{\hbar \epsilon_{0}} \mathbf{d} \cdot \operatorname{Im}\left\{\mathbf{G}_{\mathrm{c}}\left(\mathbf{r}_{0}, \mathbf{r}_{\mathbf{0}}, \omega\right)\right\} \cdot \mathbf{d}
$$

If the emitter is in a homogeneous medium, then

$$
\begin{aligned}
\Gamma_{0}\left(\mathbf{r}_{0}, \omega\right) & =\frac{2}{\hbar \epsilon_{0}} \mathbf{d} \cdot \operatorname{Im}\left\{\mathbf{G}^{\mathrm{B}}\left(\mathbf{r}_{0}, \mathbf{r}_{0}, \omega\right)\right\} \cdot \mathbf{d} \\
& =\frac{\omega^{3} n_{\mathrm{B}} \mathrm{d}^{2}}{3 \pi \epsilon_{0} \hbar c^{3}}
\end{aligned}
$$

where $\operatorname{Im}\left\{\mathbf{G}^{\mathrm{B}}\left(\mathbf{r}_{0}, \mathbf{r}_{0}, \omega\right)\right\}=\left(\omega^{3} n_{\mathrm{B}} / 6 \pi c^{3}\right) \mathbf{1}$, and $n_{\mathrm{B}}$ is the background refractive index. Thus the generalized Purcell factor is ${ }^{16,41}$

$$
\begin{aligned}
F_{\mathrm{P}}^{\mathrm{QNM}}\left(\mathbf{r}_{0}, \omega\right) & =1+\frac{\Gamma\left(\mathbf{r}_{0}, \omega\right)}{\Gamma_{0}\left(\mathbf{r}_{0}, \omega\right)} \\
& =1+\frac{\mathbf{n}_{\mathrm{d}} \cdot \operatorname{Im}\left\{\mathbf{G}_{\mathrm{c}}\left(\mathbf{r}_{0}, \mathbf{r}_{0}, \omega\right)\right\} \cdot \mathbf{n}_{\mathrm{d}}}{\mathbf{n}_{\mathrm{d}} \cdot \operatorname{Im}\left\{\mathbf{G}^{\mathrm{B}}\left(\mathbf{r}_{0}, \mathbf{r}_{0}, \omega\right)\right\} \cdot \mathbf{n}_{\mathrm{d}}} \\
& =1+\frac{6 \pi c^{3}}{\omega^{3} n_{\mathrm{B}}} \mathbf{n}_{\mathrm{d}} \cdot \operatorname{Im}\left\{\mathbf{G}_{\mathrm{c}}\left(\mathbf{r}_{0}, \mathbf{r}_{0}, \omega\right)\right\} \cdot \mathbf{n}_{\mathrm{d}}
\end{aligned}
$$

Note that we have added the extra factor of 1 , which can be derived from a Dyson equation scattering problem for dipole located outside the resonator (essentially the contribution from the homogeneous radiation modes $)^{28}$. The actual QNM contribution here is thus the modification to unity.

One can also use the QNMs to calculate the modal nonradiative decay rate of the same dipole emitter ${ }^{29,41}$, from

$$
\Gamma^{\mathrm{nrad}}\left(\mathbf{r}_{0}, \omega\right)=\frac{2}{\hbar \omega \epsilon_{0}} \int_{\mathrm{V}} \operatorname{Re}\left\{\mathbf{j}(\mathbf{r}) \cdot \mathbf{E}^{*}(\mathbf{r})\right\} d \mathbf{r}
$$


where $\mathbf{E}(\mathbf{r})=\mathbf{G}_{\mathrm{c}}\left(\mathbf{r}, \mathbf{r}_{0}, \omega\right) \cdot \frac{\mathbf{d}}{\epsilon_{0}}$ is the field of the dipole emitter, and $\mathbf{j}(\mathbf{r})=\epsilon_{0} \omega \operatorname{Im}\{\epsilon(\mathbf{r})\} \mathbf{E}(\mathbf{r})$ represents the dipole induced current density inside metal. Therefore, the nonradiative and radiative $\beta$ factor can be defined as

$$
\beta_{\mathrm{QNM}}^{\mathrm{nrad}}\left(\mathbf{r}_{0}, \omega\right)=\frac{\Gamma^{\mathrm{nrad}}\left(\mathbf{r}_{0}, \omega\right)}{\Gamma\left(\mathbf{r}_{0}, \omega\right)},
$$

and

$$
\beta_{\mathrm{QNM}}^{\mathrm{rad}}\left(\mathbf{r}_{0}, \omega\right)=1-\beta_{\mathrm{QNM}}^{\mathrm{nrad}}\left(\mathbf{r}_{0}, \omega\right)=1-\frac{\Gamma^{\mathrm{nrad}}\left(\mathbf{r}_{0}, \omega\right)}{\Gamma\left(\mathbf{r}_{0}, \omega\right)} .
$$

Impoartantly, these modal beta factors are associated with the QNM of interest, and define the probability that an emitted photon through the QNM will decay radiatively $\left(\beta_{\mathrm{QNM}}^{\mathrm{rad}}\right)$ or decay into heating ( $\left.\beta_{\mathrm{QNM}}^{\mathrm{nrad}}\right)$.

If several QNMs contribute in the spectral region of interest, then we rewrite Eq. (3) as

$$
\mathbf{G}\left(\mathbf{r}, \mathbf{r}_{0}, \omega\right)=\sum_{\mu} \mathbf{G}_{\mu}\left(\mathbf{r}, \mathbf{r}_{0}, \omega\right),
$$

where $\mathbf{G}_{\mu}\left(\mathbf{r}, \mathbf{r}_{0}, \omega\right)=A_{\mu}(\omega) \tilde{\mathbf{f}}_{\mu}(\mathbf{r}) \tilde{\mathbf{f}}_{\mu}\left(\mathbf{r}_{0}\right)$. Thus, the total decay rate of a dipole emitter is

$$
\begin{aligned}
\Gamma_{\text {total }}\left(\mathbf{r}_{0}, \omega\right) & =\frac{2}{\hbar \epsilon_{0}} \mathbf{d} \cdot \operatorname{Im}\left\{\mathbf{G}\left(\mathbf{r}_{0}, \mathbf{r}_{\mathbf{0}}, \omega\right)\right\} \cdot \mathbf{d}, \\
& =\sum_{\mu} \frac{2}{\hbar \epsilon_{0}} \mathbf{d} \cdot \operatorname{Im}\left\{\mathbf{G}_{\mu}\left(\mathbf{r}_{0}, \mathbf{r}_{\mathbf{0}}, \omega\right)\right\} \cdot \mathbf{d},
\end{aligned}
$$

and the total generalized Purcell factor is

$$
\begin{aligned}
& F_{\text {total }}^{\mathrm{QNM}}\left(\mathbf{r}_{0}, \omega\right)=1+\frac{\Gamma_{\text {total }}\left(\mathbf{r}_{0}, \omega\right)}{\Gamma_{0}\left(\mathbf{r}_{0}, \omega\right)} \\
& \quad=1+\frac{\sum_{\mu} \mathbf{n}_{\mathrm{d}} \cdot \operatorname{Im}\left\{\mathbf{G}_{\mu}\left(\mathbf{r}_{0}, \mathbf{r}_{0}, \omega\right)\right\} \cdot \mathbf{n}_{\mathrm{d}}}{\mathbf{n}_{\mathrm{d}} \cdot \operatorname{Im}\left\{\mathbf{G}^{\mathrm{B}}\left(\mathbf{r}_{0}, \mathbf{r}_{0}, \omega\right)\right\} \cdot \mathbf{n}_{\mathrm{d}}} \\
& \quad=1+\sum_{\mu} \frac{6 \pi c^{3}}{\omega^{3} n_{\mathrm{B}}} \mathbf{n}_{\mathrm{d}} \cdot \operatorname{Im}\left\{\mathbf{G}_{\mu}\left(\mathbf{r}_{0}, \mathbf{r}_{0}, \omega\right)\right\} \cdot \mathbf{n}_{\mathrm{d}} .
\end{aligned}
$$

It is important to note that the total QNM decay rates contain both radiative and nonradiative contributions. The nonradiative decay rate $^{29}$ is

$$
\Gamma_{\text {total }}^{\mathrm{nrad}}\left(\mathbf{r}_{0}, \omega\right)=\frac{2}{\hbar \omega \epsilon_{0}} \int_{\mathrm{V}} \operatorname{Re}\left\{\mathbf{j}_{\text {total }}(\mathbf{r}) \cdot \mathbf{E}_{\text {total }}^{*}(\mathbf{r})\right\} d \mathbf{r},
$$

where $\mathbf{E}_{\text {total }}(\mathbf{r})=\sum_{\mu} \mathbf{G}_{\mu}\left(\mathbf{r}, \mathbf{r}_{0}, \omega\right) \cdot \frac{\mathbf{d}}{\epsilon_{0}}$ is the total field of the dipole emitter, and $\mathbf{j}_{\text {total }}(\mathbf{r})=\epsilon_{0} \omega \operatorname{Im}\{\epsilon(\mathbf{r})\} \mathbf{E}_{\text {total }}(\mathbf{r})$ represents the dipole induced total current density inside metal. Thus, the total nonradiative and total radiative $\beta$ factor can be defined as

$$
\begin{aligned}
\beta_{\text {total }}^{\text {nrad }}\left(\mathbf{r}_{0}, \omega\right) & =\frac{\Gamma_{\text {total }}^{\mathrm{nrad}}\left(\mathbf{r}_{0}, \omega\right)}{\Gamma_{\text {total }}\left(\mathbf{r}_{0}, \omega\right)}, \\
\beta_{\text {total }}^{\mathrm{rad}}\left(\mathbf{r}_{0}, \omega\right)= & 1-\beta_{\text {total }}^{\mathrm{nrad}}\left(\mathbf{r}_{0}, \omega\right) \\
= & 1-\frac{\Gamma_{\text {total }}^{\mathrm{nrad}}\left(\mathbf{r}_{0}, \omega\right)}{\Gamma_{\text {total }}\left(\mathbf{r}_{0}, \omega\right)} .
\end{aligned}
$$

In the limit of a single mode, these beta factors define the single QNM beta factors.

\section{B. Regularized QNM fields, $\tilde{\mathbf{F}}_{\mathrm{c}}(\mathbf{R}, \omega)$, from the Dyson equation}

As mentioned in the introduction, one critical feature of the QNM field, $\tilde{\mathbf{f}}_{\mu}(\mathbf{r})$, is that it diverges at locations outside the resonator ${ }^{28,42}$, and is not convenient, neither classically nor in quantum optics. One way to rectify this problem, for arbitrarily shaped resonators, is to employ the Dyson equation to reconstruct a regularized QNM field outside the resonator, given by Eq. (1), which we repeat here for clarity: $\tilde{\mathbf{F}}_{\mu}(\mathbf{R}, \omega)=$ $\int_{V} d \mathbf{r G}^{\mathrm{B}}(\mathbf{R}, \mathbf{r}, \omega) \Delta \epsilon(\mathbf{r}, \omega) \tilde{\mathbf{f}}_{\mu}(\mathbf{r})$. In this way, one is only using the QNM within the resonator. Note that one can also use the same Dyson equation to compute the actual QNMs outside, by replacing the real frequency with the complex QNM pole frequency $\tilde{\omega}_{\mu}{ }^{15,42}$ :

$$
\tilde{\mathbf{f}}_{\mu}(\mathbf{R})=\int_{V} d \mathbf{r} \mathbf{G}^{\mathrm{B}}\left(\mathbf{R}, \mathbf{r}, \tilde{\omega}_{\mu}\right) \Delta \epsilon\left(\mathbf{r}, \tilde{\omega}_{\mu}\right) \tilde{\mathbf{f}}_{\mu}(\mathbf{r}),
$$

which in certain cases can considerably simplify the computational process of obtaining the QNMs over a wide spatial range.

Although mathematically intuitive, and convenient for obtaining the renormalized fields as certain locations, the Dyson approach to obtain a range of spatial points (e.g., $\mathbf{R}$ on a surface) is far from trivial, and can be very complex and time consuming. This is because a full 3D spatial integral is required for every single spatial point $(\mathbf{R})$ and also for a single frequency. For many problems, especially in quantum optics, one requires a full surface of points, and fields that are computed over a wide range of frequencies ${ }^{33,38}$. To exemplify the computational complexity for nanostructures, if the 3D spatial grid size of $0.2(0.5) \mathrm{nm}$ is chosen in the volume integral, then it will take approximately $\approx 8.5-10(0.6-0.7)$ minutes to obtain $\tilde{\mathbf{F}}_{\mu}$ at a single point $\mathbf{R}$ and single frequency; if the grid size is $0.1 \mathrm{~nm}$ (typical for metal nanoparticles), it will take $\approx 75-80$ minutes, also for a single point. These numbers are for a high performance workstation with 256 GB RAM, using Matlab. Obtaining these points over a closed surface (which is required to compute the quantum optical parameters, as discussed below) becomes extremely time demanding, and easily the most difficult part of the numerical calculation. Thus there is a pressing need to develop a more efficient way to obtain these regularized QNM fields, since they are required as input to quantized QNMs, and form the only reliable way to obtain meaningful modal fields outside the resonator.

\section{Regularized QNM fields from a Near-Field to Far-Field transformation}

An alternative method to obtain $\tilde{\mathbf{F}}(\mathbf{R}, \omega)$ is to perform a NF2FF transformation ${ }^{36}$, using the QNM field $\tilde{\mathbf{f}}$ at a surface close to the resonator. Near field to far field transformations are frequently exploited in antenna 
theory to obtain the far field radiation, but without the computational burden of including such fields in the main calculation (e.g., the near field solution requires a full numerical simulation, but the propagation to the far field can be done analytically). We adopt such an idea here to transform QNM fields into the desired regularized fields outside the scattering geometry. As shown in Fig. 1, we choose a cuboid surface close to resonator, as the near field $(\tilde{\mathbf{f}})$ surface, and transform to a cylindrical surface away from resonator as far field $(\tilde{\mathbf{F}})$ surface. The distances to the near and far field surface from the surface of the shown $\mathrm{Au}$ dimer are $h$ and $h_{\mathrm{far}}$. In this way, using a field equivalence principle ${ }^{43}$, i.e., the sources inside a domain are replaced with sources on the surface of this domain, and the fields inside the domain is zero, we can use the QNM with a complex frequency and transform it to a real frequency field, which has the correct far field behavior; note, the near fields are not appropriate for the evaluation of the total contribution from the radiative reservoir fields, which should be evaluated in the far field, and in real frequency space.

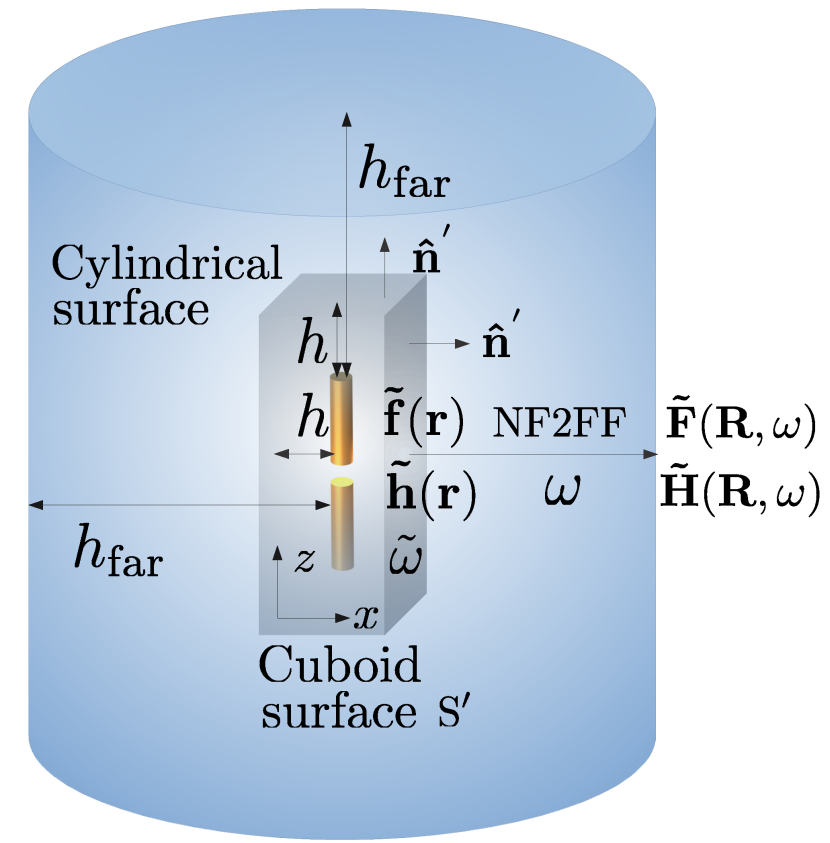

Figure 1. Schematic of NF2FF transformation. The origin of the coordinate axis is at the center of Au dimer gap. A cuboid surface and a cylindrical surface are chosen as near field surface and far field surface separately. The labels $h$ and $h_{\text {far }}$ represent the distances between them and the surface of the resonator (Au dimer). $\hat{\mathbf{n}}^{\prime}$ is a unit vector normal to near field surface, pointing outward. Using this transformation with real frequency $\omega$, one could obtain $\tilde{\mathbf{F}}_{\mathrm{c}}(\mathbf{R}, \omega)$ and $\tilde{\mathbf{H}}_{\mathrm{c}}(\mathbf{R}, \omega)$ at a far field surface from $\tilde{\mathbf{f}}_{\mathrm{c}}(\mathbf{r})$ and $\tilde{\mathbf{h}}_{\mathrm{c}}(\mathbf{r})$ obtained from the near field surfaces.

The electric surface current $\tilde{\mathbf{J}}_{\mathrm{S}^{\prime}}\left(\mathbf{r}^{\prime}\right)$ and magnetic surface current $\tilde{\mathbf{M}}_{\mathbf{S}^{\prime}}\left(\mathbf{r}^{\prime}\right)$ on a near field surface $\mathbf{S}^{\prime}$, close to resonator, are given by ${ }^{36}$

$$
\begin{array}{r}
\tilde{\mathbf{J}}_{\mathrm{S}^{\prime}}\left(\mathbf{r}^{\prime}\right)=\hat{\mathbf{n}}^{\prime} \times \tilde{\mathbf{h}}\left(\mathbf{r}^{\prime}\right), \\
\tilde{\mathbf{M}}_{\mathrm{S}^{\prime}}\left(\mathbf{r}^{\prime}\right)=-\hat{\mathbf{n}}^{\prime} \times \tilde{\mathbf{f}}\left(\mathbf{r}^{\prime}\right),
\end{array}
$$

where

$$
\tilde{\mathbf{h}}_{\mu}\left(\mathbf{r}^{\prime}\right)=\frac{1}{i \tilde{\omega}_{\mu} \mu_{0}} \nabla \times \tilde{\mathbf{f}}_{\mu}\left(\mathbf{r}^{\prime}\right),
$$

are the magnetic QNMs and $\hat{\mathbf{n}}^{\prime}$ is normal to the surface $\mathrm{S}^{\prime}$, pointing outward. Assuming the resonator is placed in a homogeneous medium with dielectric constant $\epsilon_{\mathrm{B}}=n_{\mathrm{B}}^{2}$, the QNMs fields $\tilde{\mathbf{f}} \equiv \tilde{\mathbf{f}}_{\mu}$, and magnetic QNMs are $\tilde{\mathbf{h}} \equiv \tilde{\mathbf{h}}_{\mu}$, the vector potentials at $\mathbf{R}$ generated by the electric and magnetic currents at some area element $\mathrm{dS}^{\prime}$, are

$$
\begin{array}{r}
\tilde{\mathbf{X}}(\mathbf{R}, \omega)=\frac{\epsilon_{\mathrm{B}} \epsilon_{0}}{4 \pi} \frac{e^{i k\left|\mathbf{R}-\mathbf{r}^{\prime}\right|}}{\left|\mathbf{R}-\mathbf{r}^{\prime}\right|} \tilde{\mathbf{M}}_{\mathrm{S}^{\prime}}\left(\mathbf{r}^{\prime}\right) \mathrm{d} \mathrm{S}^{\prime}, \\
\tilde{\mathbf{A}}(\mathbf{R}, \omega)=\frac{\mu_{0}}{4 \pi} \frac{e^{i k\left|\mathbf{R}-\mathbf{r}^{\prime}\right|}}{\left|\mathbf{R}-\mathbf{r}^{\prime}\right|} \tilde{\mathbf{J}}_{\mathrm{S}^{\prime}}\left(\mathbf{r}^{\prime}\right) \mathrm{d} \mathrm{S}^{\prime} .
\end{array}
$$

Thus, integrating the contributions from all the point sources distributed over the surface $\mathrm{S}^{\prime}$, we obtain the total vector potential fields ${ }^{36}$

$$
\begin{array}{r}
\tilde{\mathbf{X}}(\mathbf{R}, \omega)=\frac{\epsilon_{\mathrm{B}} \epsilon_{0}}{4 \pi} \oint_{S^{\prime}} \frac{e^{i k\left|\mathbf{R}-\mathbf{r}^{\prime}\right|}}{\left|\mathbf{R}-\mathbf{r}^{\prime}\right|} \tilde{\mathbf{M}}_{\mathrm{S}^{\prime}}\left(\mathbf{r}^{\prime}\right) \mathrm{dS}^{\prime} \\
\tilde{\mathbf{A}}(\mathbf{R}, \omega)=\frac{\mu_{0}}{4 \pi} \oint_{S^{\prime}} \frac{e^{i k\left|\mathbf{R}-\mathbf{r}^{\prime}\right|}}{\left|\mathbf{R}-\mathbf{r}^{\prime}\right|} \tilde{\mathbf{J}}_{\mathrm{S}^{\prime}}\left(\mathbf{r}^{\prime}\right) \mathrm{d} \mathrm{S}^{\prime}
\end{array}
$$

Subsequently, the regularized electric QNMs $\tilde{\mathbf{F}}$ and magnetic QNMs $\tilde{\mathbf{H}}$ in the far field are obtained from ${ }^{36,43}$

$$
\begin{aligned}
\tilde{\mathbf{F}}(\mathbf{R}, \omega)= & i \omega\left[\tilde{\mathbf{A}}(\mathbf{R}, \omega)+\frac{c^{2}}{\left(\omega n_{\mathrm{B}}\right)^{2}} \nabla(\nabla \cdot \tilde{\mathbf{A}}(\mathbf{R}, \omega))\right] \\
& -\frac{1}{\epsilon_{\mathrm{B}} \epsilon_{0}} \nabla \times \tilde{\mathbf{X}}(\mathbf{R}, \omega), \\
\tilde{\mathbf{H}}(\mathbf{R}, \omega)= & i \omega\left[\tilde{\mathbf{X}}(\mathbf{R}, \omega)+\frac{c^{2}}{\left(\omega n_{\mathrm{B}}\right)^{2}} \nabla(\nabla \cdot \tilde{\mathbf{X}}(\mathbf{R}, \omega))\right] \\
& +\frac{1}{\mu_{0}} \nabla \times \tilde{\mathbf{A}}(\mathbf{R}, \omega) .
\end{aligned}
$$

Mathematically, it is also useful to consider the fields in the very far spatial domain $\left(\mathbf{R} \rightarrow \mathbf{R}_{\infty}\right)$, since one can perform the far field approximation to obtain the simplified form for the reservoir field expression:

$$
\begin{gathered}
\tilde{\mathbf{F}}\left(\mathbf{R}_{\infty}, \omega\right) \approx i \omega\left(\tilde{\mathbf{A}}\left(\mathbf{R}_{\infty}, \omega\right)-\hat{\mathbf{R}}_{\infty}\left(\hat{\mathbf{R}}_{\infty} \cdot \tilde{\mathbf{A}}\left(\mathbf{R}_{\infty}, \omega\right)\right)\right. \\
\left.-\eta_{\alpha}^{\mathrm{B}}(\omega) \hat{\mathbf{R}}_{\infty} \times \tilde{\mathbf{X}}\left(\mathbf{R}_{\infty}, \omega\right)\right)
\end{gathered}
$$


with $\eta_{\alpha}^{\mathrm{B}}(\omega)=\sqrt{\mu_{0} / \epsilon_{0} \epsilon_{\alpha}^{\mathrm{B}}(\omega)}, \hat{\mathbf{R}}=\mathbf{R} /|\mathbf{R}|$ and the approximated vector potentials

$$
\begin{aligned}
& \tilde{\mathbf{A}}\left(\mathbf{R}_{\infty}, \omega\right) \approx \mu_{0} \frac{e^{i k\left|\mathbf{R}_{\infty}\right|}}{4 \pi\left|\mathbf{R}_{\infty}\right|} \oint_{S^{\prime}} \mathrm{d} S^{\prime} e^{-i k \hat{\mathbf{R}}_{\infty} \cdot \mathbf{r}_{s}^{\prime}} \tilde{\mathbf{J}}\left(\mathbf{r}_{s}^{\prime}\right) \\
& \tilde{\mathbf{X}}\left(\mathbf{R}_{\infty}, \omega\right) \approx \epsilon_{0} \epsilon^{\mathrm{B}} \frac{e^{i k\left|\mathbf{R}_{\infty}\right|}}{4 \pi\left|\mathbf{R}_{\infty}\right|} \oint_{S^{\prime}} \mathrm{d} S^{\prime} e^{-i k \hat{\mathbf{R}}_{\infty} \cdot \mathbf{r}_{s}^{\prime}} \tilde{\mathbf{M}}\left(\mathbf{r}_{s}^{\prime}\right)
\end{aligned}
$$

As we will show below, these considerably simplify the problem of having to integrate the fields over a closed surface, which is required to obtain the radiative coupling parameters for quantized QNM theory ${ }^{33}$.

\section{Quantized quasinormal mode parameters: quantum mechanical " $S$ factors" for Fock space normalization}

To connect the developed NF2FF to the basic formalism of a recently developed quantized QNM theory ${ }^{33,38}$, the basic theory of the quantized QNM approach is briefly recapitulated. In particular, we will show what QNM fields are required for the formalism and the related calculation of the quantum parameters required to represent the fields.

As shown in Refs. 33 and 38, combining a well established quantization approach for general absorptive and spatial inhomogeneous media ${ }^{44,45}$ with the QNM Green function, Eq. (3), one can derive an expansion of the medium-assisted electric field operator $\hat{\mathbf{E}}\left(\mathbf{r}_{\mathrm{S}}\right)$ at position $\mathbf{r}_{\mathrm{s}}$ (system region):

$$
\hat{\mathbf{E}}\left(\mathbf{r}_{\mathrm{s}}\right)=\sum_{\mu} i \sqrt{\frac{\hbar \omega_{\mu}}{2 \epsilon_{0}}} \tilde{\mathbf{f}}_{\mu}^{s}\left(\mathbf{r}_{\mathrm{s}}\right) \hat{a}_{\mu}+\text { H.a. }
$$

with the symmetrized QNM functions,

$$
\tilde{\mathbf{f}}_{\mu}^{s}\left(\mathbf{r}_{\mathrm{s}}\right)=\sum_{\nu}\left(\mathbf{S}^{\frac{1}{2}}\right)_{\nu \mu} \sqrt{\omega_{\nu} / \omega_{\mu}} \tilde{\mathbf{f}}_{\nu}\left(\mathbf{r}_{\mathrm{S}}\right),
$$

where $\hat{a}_{\mu}$ and $\hat{a}_{\mu}^{\dagger}$ are suitable annihilation and creation operators to obtain plasmon/photon Fock states for the symmetrized QNMs.

The quantum $S$ factors, which become a photon coupling matrix $S_{\mu \eta}$ if more than one QNM is considered, are given via

$$
\begin{aligned}
S_{\mu \eta} & =\int_{0}^{\infty} \mathrm{d} \omega \frac{2 A_{\mu}(\omega) A_{\eta}^{*}(\omega)}{\pi \sqrt{\omega_{\mu} \omega_{\eta}}}\left[S_{\mu \eta}^{\mathrm{nrad}}(\omega)+S_{\mu \eta}^{\mathrm{rad}}(\omega)\right], \\
& \equiv S_{\mu \eta}^{\mathrm{nrad}}+S_{\mu \eta}^{\mathrm{rad}},
\end{aligned}
$$

where

$$
S_{\mu \eta}^{\operatorname{nrad}}(\omega)=\int_{V} \mathrm{~d} \mathbf{r} \epsilon_{I}(\mathbf{r}, \omega) \tilde{\mathbf{f}}_{\mu}(\mathbf{r}) \cdot \tilde{\mathbf{f}}_{\eta}^{*}(\mathbf{r}),
$$

accounts for absorption due to the metallic losses, and

$$
S_{\mu \eta}^{\mathrm{rad}}(\omega)=\frac{1}{2 \epsilon_{0} \omega} \int_{S_{\mathrm{V}}} \mathrm{d} A_{\mathrm{s}} \hat{\mathbf{n}}_{\mathrm{s}} \cdot\left(\tilde{\mathbf{F}}_{\mu}(\mathbf{s}, \omega) \times \tilde{\mathbf{H}}_{\eta}^{*}(\mathbf{s}, \omega)+\underset{(\mu \leftrightarrow \eta)}{\text { H.c. })},\right.
$$

describes radiation leaving the system through the surface $S_{V}$ with the normal vector $\hat{\mathbf{n}}_{\mathbf{s}}$ pointing outward from the resonator volume $V$, and $\tilde{\mathbf{H}}(\mathbf{s}, \omega)=1 /\left(i \mu_{0} \omega\right) \nabla \times$ $\tilde{\mathbf{F}}(\mathbf{s}, \omega)$ is the QNM magnetic field. Furthermore, by choosing $S_{V}$ in the very far field at $S_{\infty}$, we can apply the Silver-Müller radiation condition $\hat{\mathbf{n}}_{s} \times \mathbf{H}(\mathbf{s}, \omega) \rightarrow$ $-n_{\mathrm{B}} c \epsilon_{0} \tilde{\mathbf{F}}(\mathbf{s}, \omega)$ and in addition use the approximated version of the NF2FF results (Eqs.(30)-(32)); we then arrive at an approximated formula for the radiative contribution

$$
S_{\mu \eta}^{\mathrm{rad}}(\omega) \approx \frac{n_{\mathrm{B}} c}{\omega} \int_{S_{\infty}} d A_{\mathrm{s}} \tilde{\mathbf{F}}_{\mu}\left(\mathbf{s}_{\infty}, \omega\right) \cdot \tilde{\mathbf{F}}_{\eta}^{*}\left(\mathbf{s}_{\infty}, \omega\right) .
$$

Choosing $S_{\infty}$ as a sphere and transforming into spherical coordinates, leads to a further simplification

$$
S_{\mu \eta}^{\mathrm{rad}}(\omega)=\frac{n_{\mathrm{B}} c}{\omega} I_{\mu \eta}^{\mathrm{sur}}(\omega)
$$

with

$$
\begin{aligned}
I_{\mu \eta}^{\operatorname{sur}}(\omega)= & \frac{1}{16 \pi^{2}} \int_{0}^{2 \pi} \mathrm{d} \varphi \int_{0}^{\pi} \mathrm{d} \vartheta \sin (\vartheta) \times \\
& \tilde{\mathbf{Z}}_{\mu}(\varphi, \vartheta, \omega) \cdot \tilde{\mathbf{Z}}_{\eta}^{*}(\varphi, \vartheta, \omega),
\end{aligned}
$$

where the function $\tilde{\mathbf{Z}}_{\mu}(\varphi, \vartheta, \omega)$ is given as

$$
\begin{aligned}
\tilde{\mathbf{Z}}_{\mu}(\varphi, \vartheta, \omega) & =i \omega \mu_{0} \oint_{S^{\prime}} \mathrm{d} S^{\prime} e^{-i n_{\mathrm{B}} \omega \hat{\mathbf{R}} \cdot \mathbf{r}_{s}^{\prime} / c} \\
& {\left[\tilde{\mathbf{J}}_{\mu}\left(\mathbf{r}_{s}^{\prime}\right)-\left(\tilde{\mathbf{J}}_{\mu}\left(\mathbf{r}_{s}^{\prime}\right) \cdot \hat{\mathbf{R}}\right) \hat{\mathbf{R}}-n_{\mathrm{B}} c \epsilon_{0} \hat{\mathbf{R}} \times \tilde{\mathbf{M}}_{\mu}\left(\mathbf{r}_{s}^{\prime}\right)\right], }
\end{aligned}
$$

and $\hat{\mathbf{R}}=\hat{\mathbf{R}}(\varphi, \vartheta)$ is the radial basis vector in spherical coordinates, namely:

$$
\hat{\mathbf{R}}=(\sin (\vartheta) \cos (\varphi), \sin (\vartheta) \sin (\varphi), \cos (\vartheta)) .
$$

Notably, the above form in Eq. (39) is independent of the radius of the sphere $S_{\infty}$, as long as it is chosen in the very far field, which significantly simplifies the numerical evaluation of the radiative part.

Equations (35-42) show how to use the NF2FF transform to model a quantum emitter coupled to the quantized QNMs. For example, placing an emitter with dipole moment $\mathbf{d}$ at $\mathbf{r}_{0}$, and assuming the bad cavity limit (i.e., a weakly coupled emitter), then the quantum SE rate, i.e., the SE rate obtained from the QNM quantization model, is ${ }^{33}$

$$
\Gamma_{\text {quan }}=\Gamma_{\text {quan }}^{\text {diag }}+\Gamma_{\text {quan }}^{\text {ndiag }}
$$


where the diagonal contribution is

$$
\Gamma_{\text {quan }}^{\text {diag }}=\sum_{\mu} S_{\mu \mu} \frac{\left|\tilde{g}_{\mu}\right|^{2} \gamma_{\mu}}{\Delta_{\mu \mu}^{2}+\gamma_{\mu}^{2}},
$$

and non-diagonal contribution is

$$
\Gamma_{\text {quan }}^{\mathrm{ndiag}}=\sum_{\mu, \eta \neq \mu} \tilde{g}_{\mu} S_{\mu \eta} \tilde{g}_{\eta}^{*} K_{\mu \eta},
$$

with

$$
K_{\mu \eta}=\frac{\left[i\left(\omega_{\mu}-\omega_{\eta}\right)+\gamma_{\mu}+\gamma_{\eta}\right]}{\left[2\left(\Delta_{\mu \mathrm{e}}-i \gamma_{\mu}\right)\left(\Delta_{\eta \mathrm{e}}+i \gamma_{\eta}\right)\right]} .
$$

Here, $\Delta_{\mu \mathrm{e}}=\omega_{\mu}-\omega_{\mathrm{e}}$ is the frequency detuning between the emitter and QNMs, and $\tilde{g}_{\mu}=\sqrt{\omega_{\mu} /\left(2 \epsilon_{0} \hbar\right)} \mathbf{d} \cdot \tilde{\mathbf{f}}_{\mu}\left(\mathbf{r}_{0}\right)$ is the emitter-QNM coupling. The total quantum Purcell factor is

$$
F_{\mathrm{P}}^{\text {quan }}=\frac{\Gamma_{\text {quan }}}{\Gamma^{0}}
$$

where $\Gamma^{0}$ is the spontaneous emission rate in a homogeneous medium.

Note we refer to Eq. (43) as the "quantum SE rate" in the sense that it is derived using a system-level quantized mode theory for the photons. In the limit of a single photon subspace, as appropriate for a SE description, we naturally expect agreement with the semiclassical theory for SE. Nevertheless, for effects beyond the single quantum regime, the quantum approach is required, so we use this label merely to label the rate that is computed using the quantized QNM theory.

For the single mode case, $S_{\mu \eta}$ becomes a simple photon normalization factor $S$, and takes the simplified form

$$
\begin{aligned}
S & =\frac{2}{\pi \omega_{\mathrm{c}}} \int_{0}^{\infty} \mathrm{d} \omega\left|A_{\mathrm{c}}(\omega)\right|^{2}\left[S^{\mathrm{nrad}}(\omega)+S^{\mathrm{rad}}(\omega)\right] \\
& \equiv S^{\mathrm{nrad}}+S^{\mathrm{rad}}
\end{aligned}
$$

where

$$
S^{\mathrm{nrad}}(\omega)=\int_{V} \mathrm{~d} \mathbf{r} \epsilon_{I}(\mathbf{r}, \omega)|\tilde{\mathbf{f}}(\mathbf{r})|^{2}
$$

and

$$
S^{\mathrm{rad}}(\omega)=\frac{1}{\epsilon_{0} \omega} \int_{S_{V}} \mathrm{~d} A_{\mathbf{s}} \mathbf{n}_{\mathbf{s}} \cdot \operatorname{Re}\left(\tilde{\mathbf{F}}(\mathbf{s}, \omega) \times \tilde{\mathbf{H}}^{*}(\mathbf{s}, \omega)\right) .
$$

Thus the full expressions of $S^{\text {nrad }}$ and $S^{\text {rad }}$ are

$$
\begin{aligned}
S^{\mathrm{nrad}} & =\frac{2}{\pi \omega_{\mathrm{c}}} \int_{0}^{\infty} d \omega\left|A_{\mathrm{c}}(\omega)\right|^{2} \int_{V} d \mathbf{r} \epsilon_{\mathrm{I}}(\mathbf{r}, \omega)\left|\tilde{\mathbf{f}}_{\mathrm{c}}(\mathbf{r})\right|^{2}, \\
& =\frac{2}{\pi \omega_{\mathrm{c}}} \int_{0}^{\infty} d \omega\left|A_{\mathrm{c}}(\omega)\right|^{2} \epsilon_{\mathrm{I}}(\omega) \int_{V} d \mathbf{r}\left|\tilde{\mathbf{f}}_{\mathrm{c}}(\mathbf{r})\right|^{2} .
\end{aligned}
$$

and

$$
\begin{aligned}
S^{\mathrm{rad}}=\frac{2}{\pi \omega_{\mathrm{c}}} \int_{0}^{\infty} d \omega\left|A_{\mathrm{c}}(\omega)\right|^{2} & \\
& \frac{1}{\epsilon_{0} \omega} \int_{S_{V}} d A_{\mathrm{s}} \mathbf{n}_{\mathrm{s}} \cdot \operatorname{Re}\left(\tilde{\mathbf{F}}(\mathbf{s}, \omega) \times \tilde{\mathbf{H}}^{*}(\mathbf{s}, \omega)\right) .
\end{aligned}
$$

Once again, choosing $S_{V}$ as sphere in the very far field at $S_{\infty}$ (and applying the same approximations as in the multi-mode case) leads to

$$
S^{\mathrm{rad}} \approx \frac{2}{\pi \omega_{\mathrm{c}}} \int_{0}^{\infty} d \omega\left|A_{\mathrm{c}}(\omega)\right|^{2} \frac{n_{\mathrm{B}} c}{\omega} I^{\mathrm{sur}}(\omega),
$$

with

$$
I^{\operatorname{sur}}(\omega)=\frac{1}{16 \pi^{2}} \int_{0}^{2 \pi} \mathrm{d} \varphi \int_{0}^{\pi} \mathrm{d} \vartheta \sin (\vartheta)\left|\tilde{\mathbf{Z}}_{\mathrm{c}}(\varphi, \vartheta, \omega)\right|^{2} .
$$

Placing an emitter with dipole moment $\mathbf{d}$ at $\mathbf{r}_{0}$, and assuming the bad cavity limit, the quantum SE rate is

$$
\Gamma_{\text {quan }, \mathrm{c}}=S \frac{\left|\tilde{g}_{\mathrm{c}}\right|^{2} \gamma_{\mathrm{c}}}{\Delta_{\mathrm{ce}}^{2}+\gamma_{\mathrm{c}}^{2}},
$$

where $\Delta_{\text {ce }}=\omega_{\mathrm{c}}-\omega_{\mathrm{e}}$ is the frequency detuning between the emitter and single QNM, and $\tilde{g}_{\mathrm{c}}=\sqrt{\omega_{\mathrm{c}} /\left(2 \epsilon_{0} \hbar\right)} \mathbf{d}$. $\tilde{\mathbf{f}}_{\mathrm{C}}\left(\mathbf{r}_{0}\right)$ is the emitter-QNM coupling. Then the quantum Purcell factor is

$$
F_{\mathrm{P}}^{\text {quan,c }}=\frac{\Gamma_{\text {quan }, \mathrm{c}}}{\Gamma^{0}} .
$$

In the limit that $S \rightarrow 1$, Eqs. (55)-(56) recover the wellknown decay rate and Purcell factor from the dissipative Jaynes-Cummings model ${ }^{46}$.

Furthermore, the radiative and non-radiative contributions are associated with the beta factors, defined also classically in Eq. (12) and Eq. (13). In the quantized QNM theory, the beta factors are defined from

$$
\begin{aligned}
& \beta_{\text {quan }}^{\mathrm{rad}}=\frac{S^{\mathrm{rad}}}{S}, \\
& \beta_{\text {quan }}^{\text {nrad }}=\frac{S^{\mathrm{nrad}}}{S} .
\end{aligned}
$$

These quantum-derived $S$ factors are unitless quantities, and for well isolated single QNMs, we have found that ${ }^{33}$ $S \approx 1$ (see also calculations below for gold dimers).

Although the Purcell factors obtained from the quantized QNM theory have been shown to be in excellent agreement with the semiclassical results (also using the QNM approximation) ${ }^{33}$, and therefore with the full Maxwell solution, there can be generally a discrepancy between both approaches; the reason for this is because different approximations to the full Green function are imposed on different stages of the derivation. Whereas in the semiclassical case, the QNM approximation is done at the emitter position $\mathbf{r}_{0}$ only, in the quantum case, the 
approximations is applied to all positions within the resonator region. This is deeply connected to the relation

$$
\begin{aligned}
\operatorname{Im} \mathbf{G}\left(\mathbf{r}_{0}, \mathbf{r}_{0}\right)= & \int_{V} d \mathbf{r} \epsilon_{I}(\mathbf{r}) \mathbf{G}\left(\mathbf{r}_{0}, \mathbf{r}\right) \cdot \mathbf{G}\left(\mathbf{r}, \mathbf{r}_{0}\right) \\
& +i \frac{c^{2}}{2 \omega^{2}} \int_{S} d A_{s} \mathbf{C}\left(\mathbf{s}, \mathbf{r}_{0}\right)-\mathbf{C}^{\dagger}\left(\mathbf{s}, \mathbf{r}_{0}\right)
\end{aligned}
$$

with

$$
\mathbf{C}\left(\mathbf{s}, \mathbf{r}_{0}\right)=\left[\nabla \times \mathbf{G}\left(\mathbf{s}, \mathbf{r}_{0}\right)\right]^{t} \cdot\left[\mathbf{n}_{s} \times \mathbf{G}^{*}\left(\mathbf{s}, \mathbf{r}_{0}\right)\right]
$$

In the semiclassical case, the QNM approximation is done on the lhs (left hand side) of (59); however, in the quantized QNM theory, the approximation must be done on the rhs in order to construct Fock states, that are independent on the emitter positions and in order to formulate the electric field operator in the cavity with few mode operators, i.e., instead of an infinite set of positiondependent operators.

So summarize this subsection, the quantum $S$ factors for quantized QNM theory are greatly desired in the process of the quantization of the open cavities, and the calculation of the related quantum quantities, such as coupling coefficient, spontaneous emission rate, Purcell factors, and single photon source figures of merit ${ }^{38}$. Thus it is important to have accurate efficient numerical techniques to obtain the required fields and integrals.

\section{E. Practical evaluation of the frequency integrals in the quantized QNM model}

Due to the introduction of a rectangular function in $A_{\mathrm{c}}(\omega)$ (Eq. (5)), the frequency integral in $S$ is restricted on a finite frequency band around the QNM center frequency $\omega_{\mathrm{c}}$. If the non-Lorentzian contributions are nearly constant in this effective frequency regime, we can approximate $S$ as

$$
\int_{0}^{\infty} d \omega S(\omega) \approx S_{\mathrm{p}}
$$

In the multi-mode case, the frequency integrals are performed, in the same approximation, as

$$
\int_{0}^{\infty} d \omega S_{\mu \eta}(\omega) \approx S_{\mathrm{p}, \mu \eta}
$$

where $S_{\mathrm{p}, \mu \eta}$ is an average of the non-modal contributions calculated at $\omega_{\mu}, \omega_{\eta}$.

Within these approximations, the pole terms of the photon coupling matrices and photon normalization factors take the form

$$
\begin{array}{rl}
S_{\mathrm{p}, \mu \eta}^{\mathrm{nrad}}=\frac{\sqrt{\omega_{\mu} \omega_{\eta}}}{i\left(\tilde{\omega}_{\mu}-\tilde{\omega}_{\eta}^{*}\right)} \int_{V} & \mathrm{~d} \mathbf{r} \sqrt{\epsilon_{I}\left(\mathbf{r}, \omega_{\mu}\right) \epsilon_{I}\left(\mathbf{r}, \omega_{\eta}\right)} \\
& \times \tilde{\mathbf{f}}_{\mu}(\mathbf{r}) \cdot \tilde{\mathbf{f}}_{\eta}(\mathbf{r})
\end{array}
$$

and

$$
\begin{aligned}
S_{\mathrm{p} 1, \mu \eta}^{\mathrm{rad}}= & \frac{1}{i\left(\tilde{\omega}_{\mu}-\tilde{\omega}_{\eta}\right) 2 \epsilon_{0} \omega} \\
& \int_{S_{\mathrm{V}}} \mathrm{d} A_{\mathrm{s}} \hat{\mathbf{n}}_{\mathrm{s}} \cdot\left(\tilde{\mathbf{F}}_{\mu}\left(\mathbf{s}, \omega_{\mu}\right) \times \tilde{\mathbf{H}}_{\eta}^{*}\left(\mathbf{s}, \omega_{\eta}\right)+\underset{(\mu \leftrightarrow \eta)}{\text { H.c. })}\right)
\end{aligned}
$$

For the latter case, we can also derive an alternative pole approximation if one is interested in the integrated value over a far field surface:

$$
S_{\mathrm{p} 2, \mu \eta}^{\mathrm{rad}}=\frac{n_{\mathrm{B}} c}{i\left(\tilde{\omega}_{\mu}-\tilde{\omega}_{\eta}\right)} I_{\mu \eta}^{\mathrm{sur}}
$$

where

$$
\begin{gathered}
I_{\mu \eta}^{\text {sur }}=\frac{1}{16 \pi^{2}} \int_{0}^{2 \pi} \mathrm{d} \varphi \int_{0}^{\pi} \mathrm{d} \vartheta \sin (\vartheta) \times \\
\tilde{\mathbf{Z}}_{\mu}\left(\varphi, \vartheta, \omega_{\mu}\right) \cdot \tilde{\mathbf{Z}}_{\eta}^{*}\left(\varphi, \vartheta, \omega_{\eta}\right) .
\end{gathered}
$$

For the single QNM case, then

$$
\begin{gathered}
S_{\mathrm{p}}^{\mathrm{nrad}}=Q \int_{V} \mathrm{~d} \mathbf{r} \epsilon_{I}\left(\mathbf{r}, \omega_{\mathrm{c}}\right)|\tilde{\mathbf{f}}(\mathbf{r})|^{2} \\
S_{\mathrm{p} 1}^{\mathrm{rad}}=\frac{1}{2 \epsilon_{0} \gamma_{\mathrm{c}}} \int_{S_{V}} d A_{\mathrm{s}} \mathbf{n}_{\mathrm{s}} \cdot \operatorname{Re}\left(\tilde{\mathbf{F}}\left(\mathbf{s}, \omega_{\mathrm{c}}\right) \times \tilde{\mathbf{H}}^{*}\left(\mathbf{s}, \omega_{\mathrm{c}}\right)\right) \\
S_{\mathrm{p} 2}^{\mathrm{rad}}=\frac{n_{\mathrm{B}} c}{2 \gamma_{\mathrm{c}}} I_{\mathrm{c}}^{\mathrm{sur}}
\end{gathered}
$$

and

$$
I_{\mathrm{c}}^{\text {sur }}=\frac{1}{16 \pi^{2}} \int_{0}^{2 \pi} \mathrm{d} \varphi \int_{0}^{\pi} \mathrm{d} \vartheta \sin (\vartheta)\left|\tilde{\mathbf{Z}}_{\mathrm{c}}\left(\varphi, \vartheta, \omega_{\mathrm{c}}\right)\right|^{2}
$$

\section{NUMERICAL RESULTS FOR SINGLE QUASINORMAL MODES OF METAL NANOROD DIMERS}

\section{A. Single quasinormal modes for metal dimers: role of material losses}

As shown in Fig. 2 (a), we first consider a gold $(\mathrm{Au})$ rod dimer (with diameter of $D_{\mathrm{Au}}=20 \mathrm{~nm}$, length of $h_{\mathrm{Au}}=80 \mathrm{~nm}$ and gap of $h_{\text {gap }}=20 \mathrm{~nm}$ ) in free space $\left(\epsilon_{\mathrm{B}}=n_{\mathrm{B}}^{2}=1.0\right)$, with the same parameters as used in Ref. 33. The local dielectric function of $\mathrm{Au}$ is described by the Drude model,

$$
\epsilon_{\mathrm{Au}}=1-\frac{\omega_{\mathrm{p}}^{2}}{\omega^{2}+i \omega \gamma_{\mathrm{p}}},
$$

where $\hbar \omega_{\mathrm{p}}=8.2934 \mathrm{eV}\left(\omega_{\mathrm{p}}=1.26 \times 10^{16} \mathrm{rad} / \mathrm{s}\right)$ and $\hbar \gamma_{\mathrm{p}}=0.0928 \mathrm{eV}\left(\gamma_{\mathrm{p}}=\gamma_{\mathrm{p} 0}=1.41 \times 10^{14} \mathrm{rad} / \mathrm{s}\right)$.

In order to understand the effect of material losses on the dimer QNMs properties, we will also artificially 


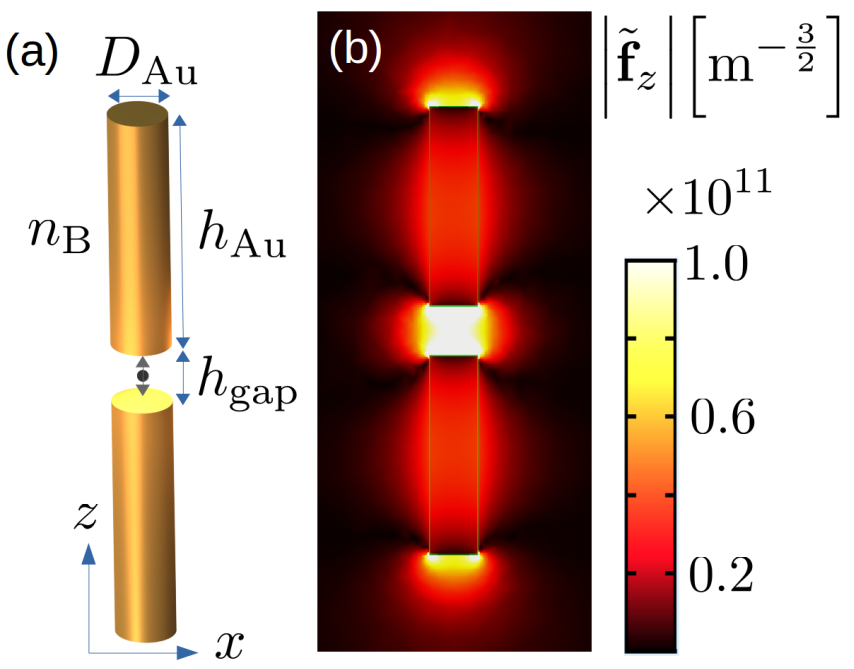

Figure 2. (a) Schematic diagram of Au dimer in free space. (b) Normalized QNM field $\left|\tilde{\mathbf{f}}_{z}\right|$ (dominant component) distribution at plane $y=0$ for $\gamma_{\mathrm{p}}=\gamma_{\mathrm{p} 0}$. Here the absolute value means that both the real and the imaginary parts are taken into account. The origin of the coordinate system is at the gap center of the dimer. The other QNMs for different loss values look similar.

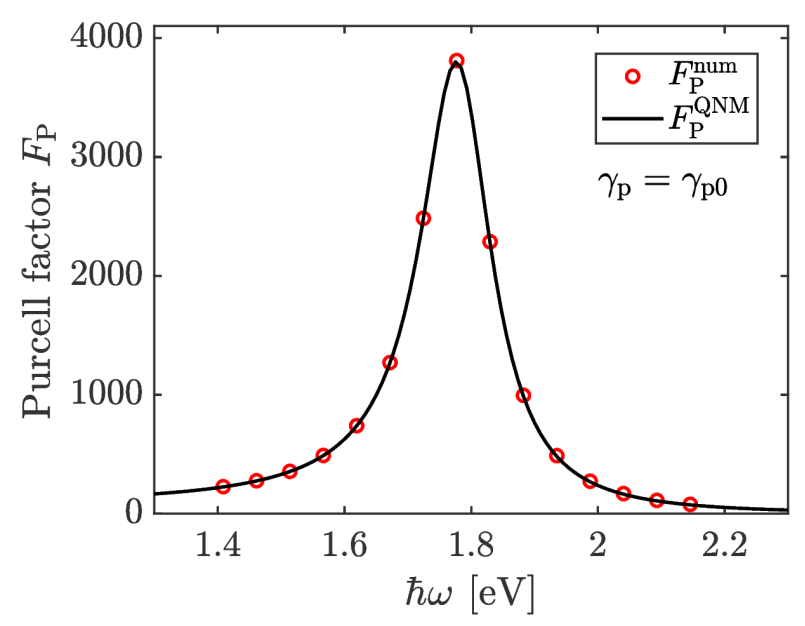

Figure 3. Classical Purcell factor calculations for a $z$-polarized dipole at dimer center, with $\gamma_{\mathrm{p}}=\gamma_{\mathrm{p} 0}$ (gold), using the analytical QNM formula (Eq. (10)) and the full dipole formula (Eq. (79)).

change the loss term $\gamma_{p}$ in the metal Drude model to $3 \gamma_{\mathrm{p} 0}, 2 \gamma_{\mathrm{p} 0},(2 / 3) \gamma_{\mathrm{p} 0}$ and $(1 / 3) \gamma_{\mathrm{p} 0}$. The corresponding complex QNM frequency $\tilde{\omega}_{\mathrm{c}}=\omega_{c}-i \gamma_{c}$, quality factor $Q_{\mathrm{c}}\left(=\omega_{\mathrm{c}} / 2 \gamma_{\mathrm{c}}\right)$, QNM field $\tilde{\mathbf{f}}_{z}\left(\mathbf{r}_{0}\right)$ (z-component) at the center of the Au dimer gap, and effective mode volume $V_{\mathrm{c}}^{\text {eff }}\left(\mathbf{r}_{0}\right)\left(V_{\mathrm{c}}^{\text {eff }}=1 / \operatorname{Re}\left[\tilde{\mathbf{f}}_{z}^{2}\left(\mathbf{r}_{0}\right)\right]\right.$ and $\left.\lambda_{\mathrm{c}}=2 \pi c / \operatorname{Re}\left(\tilde{\omega}_{\mathrm{c}}\right)\right)$ are shown in Table I. The real part of the $\tilde{\omega}_{\mathrm{c}}$ and $\tilde{\mathbf{f}}_{z}\left(\mathbf{r}_{0}\right)$ are noted to be very similar in all cases, while the quality factors $Q_{\mathrm{c}}$ increases with the decrease of $\gamma_{\mathrm{p}}$ as expected.

Using the method that Bai et al. proposed ${ }^{23}$, we can obtain the normalized QNM fields from a simple dipole excitation. Specifically, the scattered electric field of a point dipole at position $\mathbf{r}_{0}$ is related to the Green's function, and given by

$$
\mathbf{E}^{\mathrm{S}}(\mathbf{r}, \omega)=\frac{1}{\epsilon_{0}} \mathbf{G}\left(\mathbf{r}, \mathbf{r}_{0}, \omega\right) \cdot \mathbf{d},
$$

where $\mathbf{d}$ is the dipole moment of the emitter. If only a single mode is dominant, we can expand the Green's function with one QNM, so that

$$
\mathbf{E}^{\mathrm{S}}(\mathbf{r}, \omega)=\frac{1}{\epsilon_{0}} A(\omega) \tilde{\mathbf{f}}_{\mathrm{c}}(\mathbf{r}) \tilde{\mathbf{f}}_{\mathrm{c}}\left(\mathbf{r}_{0}\right) \cdot \mathbf{d}
$$

Multiplying Eq. (75) with $\mathbf{d}$ and first using $\mathbf{r}=\mathbf{r}_{0}$, then

$$
\mathbf{d} \cdot \tilde{\mathbf{f}}_{\mathrm{c}}\left(\mathbf{r}_{0}\right)=\sqrt{\frac{\epsilon_{0} \mathbf{d} \cdot \mathbf{E}^{\mathrm{s}}\left(\mathbf{r}_{0}, \omega\right)}{A(\omega)}} .
$$

Substituting this back to Eq. (75), we obtain the normalized field as a function of space

$$
\begin{aligned}
& \tilde{\mathbf{f}}_{\mathrm{c}}(\mathbf{r})=\sqrt{\frac{\epsilon_{0}}{A(\omega) \mathbf{d} \cdot \mathbf{E}^{\mathrm{s}}\left(\mathbf{r}_{0}, \omega\right)}} \mathbf{E}^{\mathrm{s}}(\mathbf{r}, \omega), \\
& =\sqrt{\frac{2 \epsilon_{0}\left(\tilde{\omega}_{\mathrm{c}}-\omega\right)}{\omega \mathbf{d} \cdot \mathbf{E}^{\mathrm{s}}\left(\mathbf{r}_{0}, \omega\right)}} \mathbf{E}^{\mathrm{s}}(\mathbf{r}, \omega),
\end{aligned}
$$

and corresponding effective mode volume is simply ${ }^{15}$ :

$$
V_{\mathrm{c}}^{\mathrm{eff}}\left(\mathbf{r}_{0}\right)=\frac{1}{\epsilon\left(\mathbf{r}_{0}\right) \operatorname{Re}\left[\mathbf{f}_{\mathrm{c}}^{2}\left(\mathbf{r}_{0}\right)\right]} .
$$

We performed the the QNM simulations in a commercial COMSOL software ${ }^{47}$, where $\omega=\left(1-10^{-5}\right) \times \tilde{\omega}_{\mathrm{c}}$, very close to the pole frequency. For the single mode case, we define $\tilde{\mathbf{f}}=\tilde{\mathbf{f}}_{\mathrm{c}}$. The computational domain (including perfectly matched layers (PMLs)) is around $3 \mu \mathrm{m}^{3}$, where the maximum mesh element sizes are $0.1 \mathrm{~nm}, 2$ $\mathrm{nm}$ and $80 \mathrm{~nm}$ at the dipole point (center of the gap), inside and outside the metal. To minimize boundary reflections, we used 5 perfectly matched layers (PMLs) with a total thickness of $300 \mathrm{~nm}$, which was found to be well converged numerically.

Table I. Single QNM resonance frequency $\tilde{\omega}_{c}$, quality factor $Q_{\mathrm{c}}$, normalized QNM field $\tilde{\mathbf{f}}_{z}$ at the center of the dimer gap, and corresponding effective mode volume for various material losses. All parameters are calculated using the classical QNM theory.

\begin{tabular}{|c|c|c|c|c|}
\hline$\gamma_{\mathrm{p}}$ & $\hbar \tilde{\omega}_{c}[\mathrm{eV}]$ & $Q_{\mathrm{c}}$ & $\tilde{\mathbf{f}}_{z}\left(\mathbf{r}_{0}\right)\left[10^{9} \cdot \mathrm{m}^{-\frac{3}{2}}\right]$ & $V_{\mathrm{c}}^{\text {eff }}\left(\mathbf{r}_{0}\right) / \lambda_{\mathrm{c}}^{3}$ \\
\hline $3 \gamma_{\mathrm{p} 0}$ & $(1.773-0.147 i)$ & 6.0 & $(106.2+i 3.287)$ & $2.594 \times 10^{-4}$ \\
\hline $2 \gamma_{\mathrm{p} 0}$ & $(1.777-0.107 i)$ & 8.3 & $(106.1+i 2.367)$ & $2.615 \times 10^{-4}$ \\
\hline$\gamma_{\mathrm{p} 0}$ & $(1.780-0.068 i)$ & 13.1 & $(106.0+i 1.451)$ & $2.631 \times 10^{-4}$ \\
\hline$\frac{2}{3} \gamma_{\mathrm{p} 0}$ & $(1.781-0.055 i)$ & 16.3 & $(106.0+i 1.146)$ & $2.636 \times 10^{-4}$ \\
\hline$\frac{1}{3} \gamma_{\mathrm{p} 0}$ & $(1.781-0.041 i)$ & 21.5 & $(106.0+i 0.842)$ & $2.640 \times 10^{-4}$ \\
\hline
\end{tabular}



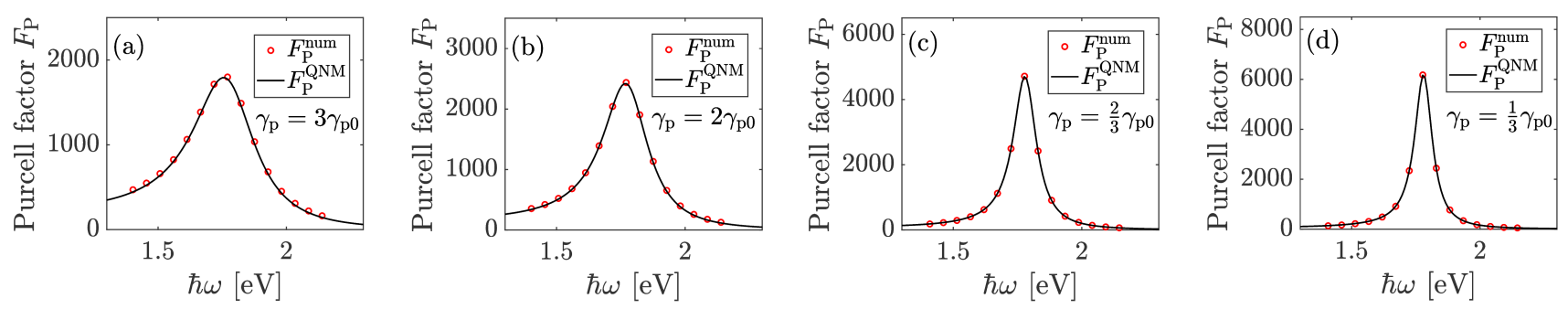

Figure 4. Classical Purcell factor calculations for a $z$-polarized point dipole placed at dimer center (full dipole result $F_{\mathrm{P}}^{\text {num }}$ from Eq. (79) versus $F_{\mathrm{P}}^{\mathrm{QNM}}$ from single QNM with Eq. (10)) for (a) $\gamma_{\mathrm{p}}=3 \gamma_{\mathrm{p} 0}$, (b) $2 \gamma_{\mathrm{p} 0}$, (c) (2/3) $\gamma_{\mathrm{p} 0}$, and (d) $(1 / 3) \gamma_{\mathrm{p} 0}$.

The numerical Purcell factors with full dipole method is defined as follows (the analytic QNM one is defined through Eq. (10) in Sec. II A):

$$
F_{\mathrm{P}}^{\text {num }}\left(\mathbf{r}_{0}, \omega\right)=\frac{\int_{\mathrm{S}} \hat{\mathbf{n}} \cdot \mathbf{S}_{\text {dipole,total }}(\mathbf{r}, \omega) d \mathrm{~A}}{\int_{\mathrm{S}} \hat{\mathbf{n}} \cdot \mathbf{S}_{\text {dipole, background }}(\mathbf{r}, \omega) d \mathrm{~A}},
$$

where $\mathrm{S}$ is a small spherical surface (with radius $1 \mathrm{~nm}$ ) surrounding dipole point and $\hat{\mathbf{n}}$ is a unit vector normal to $\mathrm{S}$, pointing outward. The vector $\mathbf{S}(\mathbf{r}, \omega)$ is the Poynting vector at this small surface and the subscript 'total' and 'background' represent the case with and without resonator. The excellent agreement with the Purcell factors using the QNM method (Eq. (10)) and full dipole method (Eq. (79)) indicate the validity of the QNM results (see Figs. 3, 4).

In addition to the full-dipole numerical Purcell factors, the numerical radiative beta factor (assuming single QNM behaviour) is defined as

$$
\beta_{\text {num }}^{\text {rad }}\left(\mathbf{r}_{0}, \omega\right)=\frac{\int_{\mathrm{S}^{\prime}} \hat{\mathbf{n}} \cdot \mathbf{S}_{\mathrm{PML}, \text { total }}\left(\mathbf{r}^{\prime}, \omega\right) d \mathrm{~A}^{\prime}}{\int_{\mathrm{S}} \hat{\mathbf{n}} \cdot \mathbf{S}_{\text {dipole,total }}(\mathbf{r}, \omega) d \mathrm{~A}},
$$

where $\mathrm{S}^{\prime}$ is the interface of PML and internal module, and $\mathbf{S}_{\mathrm{PML}, \text { total }}\left(\mathbf{r}^{\prime}, \omega\right)$ is the Poynting vector at this interface. Similarly, the numerical nonradiative beta factor is

$$
\beta_{\text {num }}^{\text {nrad }}\left(\mathbf{r}_{0}, \omega\right)=1-\beta_{\text {num }}^{\text {rad }}\left(\mathbf{r}_{0}, \omega\right) .
$$

Note that in contrast to the quantum beta factors (Eqs. (57)-(58)), the classical beta factors are frequency dependent, but are most important near $\omega_{\mathrm{c}}$.

\section{B. Calculation of the regularized QNM fields using a near-field to far-field transformation}

In order to verify the accuracy of the NF2FF transformation, and to confirm that it works correctly, we compare the $\tilde{\mathbf{F}}\left(\mathbf{R}, \omega_{\mathrm{c}}\right)$ obtained from the NF2FF transformation with the Dyson equation as selected spatial points outside the resonator, as shown in Fig. 5. As expected, in the far field zone, these fields compare extremely well.

The computational run time of the NF2FF transformation, for a spatial single point, is 0.44 minutes from the surface $h=50 \mathrm{~nm}$ (grid size $0.5 \mathrm{~nm}$ ), and 0.24 minutes using $h=30 \mathrm{~nm}$ (grid size $0.5 \mathrm{~nm}$ ). However, since some of the vector potential points needed for the transformation are also used at other points, the scaling to more points is much faster than linear. For example, using an average over 37 points, it take about 0.22 minutes per point from the surface $h=50 \mathrm{~nm}$. Using spatial points inside the resonator with a grid size of $0.2 \mathrm{~nm}$, then the Dyson equation takes about 10 minutes for a single point. In the following calculation of $S^{\mathrm{rad}}$, we need to calculate many points on a surface, as shown in Fig. 6, and we give the total run time needed for calculations using Matlab on a single computer workstation. More detailed computational run times are given later.

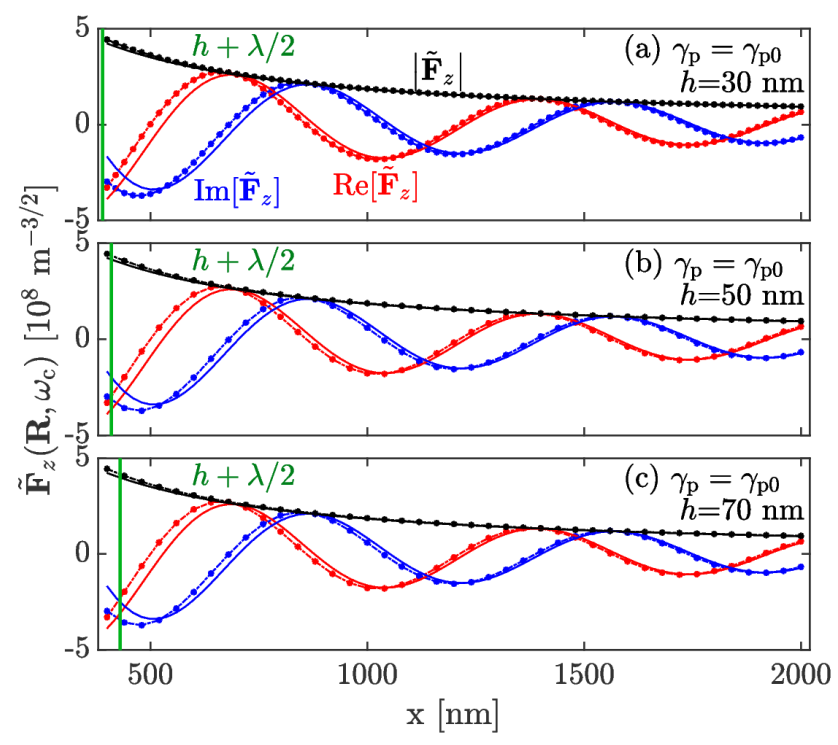

Figure 5. Comparison of $\tilde{\mathbf{F}}_{z}\left(\mathbf{R}, \omega_{\mathrm{c}}\right)$ (the real part, the imaginary part, and the absolute value) obtained from Dyson equation (solid line, Eq. (1)) and NF2FF transformation (dotted line, Eq. (28)) for $\gamma_{\mathrm{p}}=\gamma_{\mathrm{p} 0}$. Here $y=z=0$, thus $\mathbf{R}=(x, 0,0)$. The two approaches are seen to agree very well after about one wavelength outside the resonator $(\lambda)$.

Using a NF2FF transformation from a near field surface at $h=50 \mathrm{~nm}$, we also display surface plots of $\left|\operatorname{Re}\left(\tilde{\mathbf{F}}_{i}\left(\omega_{\mathrm{c}}\right)\right)\right|,\left|\operatorname{Re}\left(\tilde{\mathbf{f}}_{i}\right)\right|$ in Fig. 7 , where we show the $z$ and $x$ components at the $x z$ plane $(y=0 \mathrm{~nm})$; the $y$ component can be ignored since it is much smaller than 


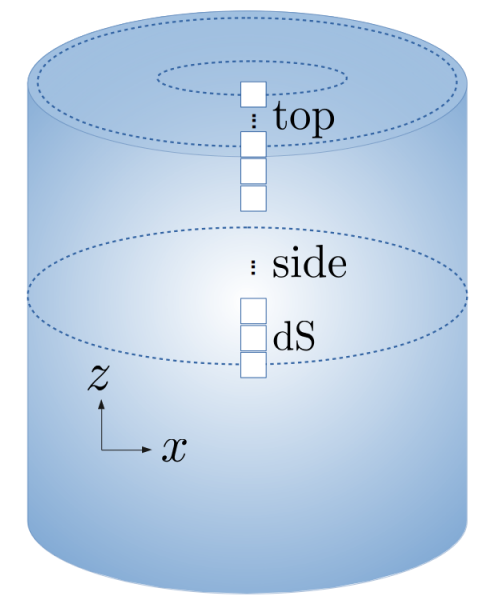

Figure 6. Schematic of the simplification that can be used for calculating $S^{\mathrm{rad}}$ (Eq. (51)) and $S_{\mathrm{p} 1}^{\mathrm{rad}}$ (Eq. (70)) using symmetry of QNM field (dimer).

other two components. To better display $z$ and $x$ components with the same scale, the $x$ component is multiplied by a factor of 2 . The ranges of $z$ and $x$ are $(-\lambda, \lambda)$ and $(h+0.5 \lambda, h+5.5 \lambda)$, where $\lambda \approx 700 \mathrm{~nm}$; $|\operatorname{Re}(\tilde{\mathbf{f}})|$ show increasing behavior (eventually divergent) while $\left|\operatorname{Re}\left(\tilde{\mathbf{F}}\left(\omega_{\mathrm{c}}\right)\right)\right|$ is convergent. Also, these fields show periodic distribution along $x$ direction with a period. Moreover, we also show $\tilde{\mathbf{F}}$ at two $y z$ surfaces with $x=400$ $\mathrm{nm}=h+0.5 \lambda$ (Fig. 8) and $x=1800 \mathrm{~nm}=h+2.5 \lambda$ (Fig. 9) for $\gamma_{\mathrm{p}}=\gamma_{\mathrm{p} 0}$ using NF2FF transformation (Eqs. (28)) from near field surface $h=50 \mathrm{~nm}$. These far field regions are directly related to the output fields that experiments can detect. However, importantly, these fields are obtained directly from the QNMs, and they also remain orthogonal to each other, which is precisely why we call them QNM regularized fields or QNM reservoir fields.

\section{Calculation of $S^{\text {nrad }}$ using the QNM fields}

To calculate $S^{\text {nrad }}$ (Eq. (51)) and $S_{\mathrm{p}}^{\text {nrad }}$ (Eq. (69)), a spatial volume integration is needed, within the metal. Below, we performed two approaches to obtain the numerical space integration: (1) the normalized QNM fields were extracted from COMSOL with some extrapolated mesh size, then the calculation was performed in Matlab; or (2) the spatial integration was performed directly in COMSOL with its own grid selection, which will be more accurate because there is no need to artificially choose the grid, minimizing interpolation errors.

As shown in Fig. 10 (a), we tested four different grid sizes; as expected, smaller grids lead to more accurate calculations and eventual convergence of the integral. If we use the second approach, we obtain $S^{\text {nrad }}=0.595$ and $S_{\mathrm{p}}^{\text {nrad }}=0.583$, which is very close to the results from first approach with a grid size of $0.1 \mathrm{~nm}$, and is also very close to the result of 0.58 , reported in Ref. 33 .

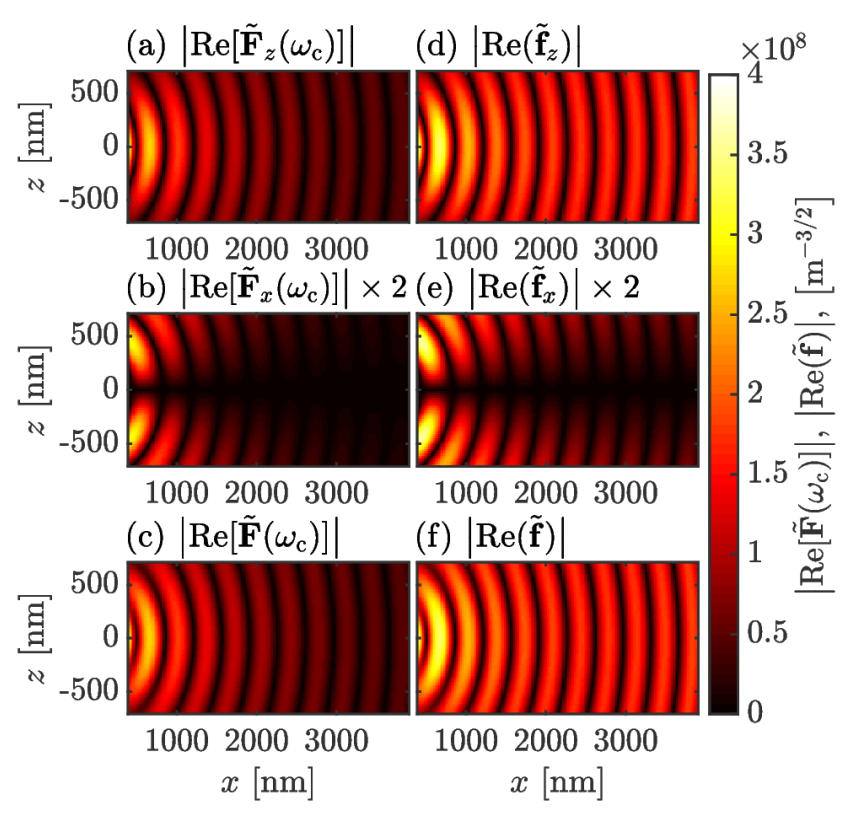

Figure 7. (a) $\left|\operatorname{Re}\left[\tilde{\mathbf{F}}_{z}\left(\omega_{\mathrm{c}}\right)\right]\right|$, (b) $2\left|\operatorname{Re}\left[\tilde{\mathbf{F}}_{x}\left(\omega_{\mathrm{c}}\right)\right]\right|$, (c) $\left|\operatorname{Re}\left[\tilde{\mathbf{F}}\left(\omega_{\mathrm{c}}\right)\right]\right|$, (d) $\left|\operatorname{Re}\left(\tilde{\mathbf{f}}_{z}\right)\right|$, (e) $2\left|\operatorname{Re}\left(\tilde{\mathbf{f}}_{x}\right)\right|$, and (f) $|\operatorname{Re}(\tilde{\mathbf{f}})|$ at plane $y=0 \mathrm{~nm}$ for $\gamma_{\mathrm{p}}=\gamma_{\mathrm{p} 0}$, using the NF2FF transformation (Eq. (28)) from near field surface $h=50 \mathrm{~nm}$. Ranges of $z$ and $x$ are $(-\lambda, \lambda)$ and $(h+0.5 \lambda, h+5.5 \lambda)$, where $\lambda \approx 700 \mathrm{~nm}$. Note the $z$-component is the dominant one, and the $y$-component can be ignored.

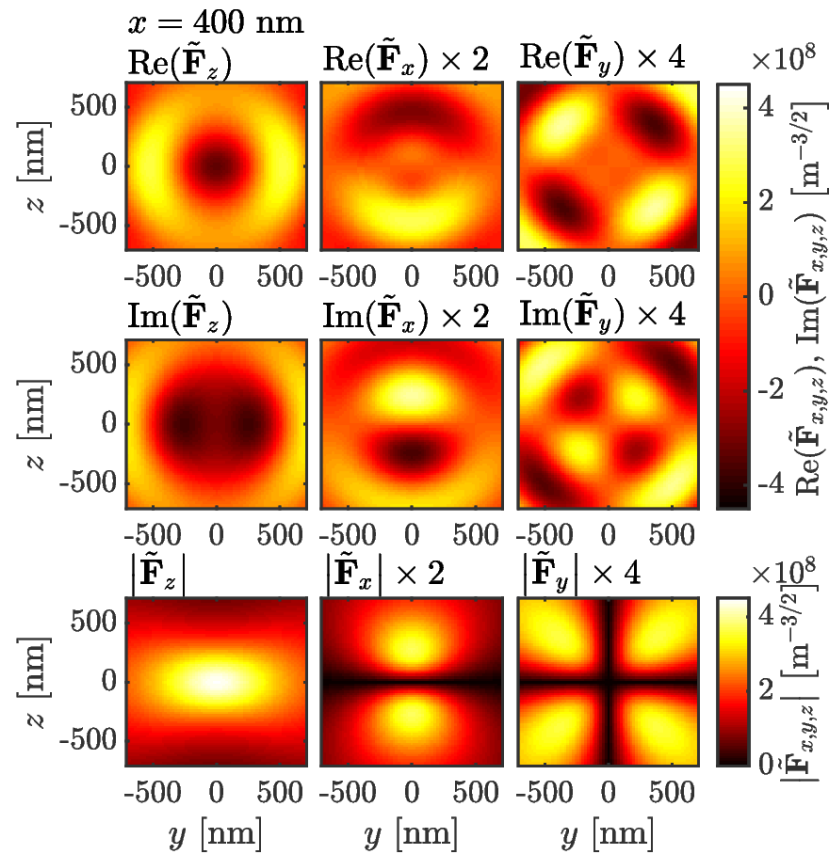

Figure 8. $\quad \tilde{\mathbf{F}}_{z}, \tilde{\mathbf{F}}_{x}$ and $\tilde{\mathbf{F}}_{y}$ at plane $x=400 \mathrm{~nm}$ for $\gamma_{\mathrm{p}}=$ $\gamma_{\mathrm{p} 0}$ using NF2FF transformation (Eqs. (28)) from near field surface $h=50 \mathrm{~nm}$. Ranges of $z$ and $x$ are $(-\lambda, \lambda)$, where $\lambda \approx 700 \mathrm{~nm}$. $z$-component is the dominant one. The $x$ and $y$ components are multiplied by a factor of 2 and 4 to better display the field distribution. 


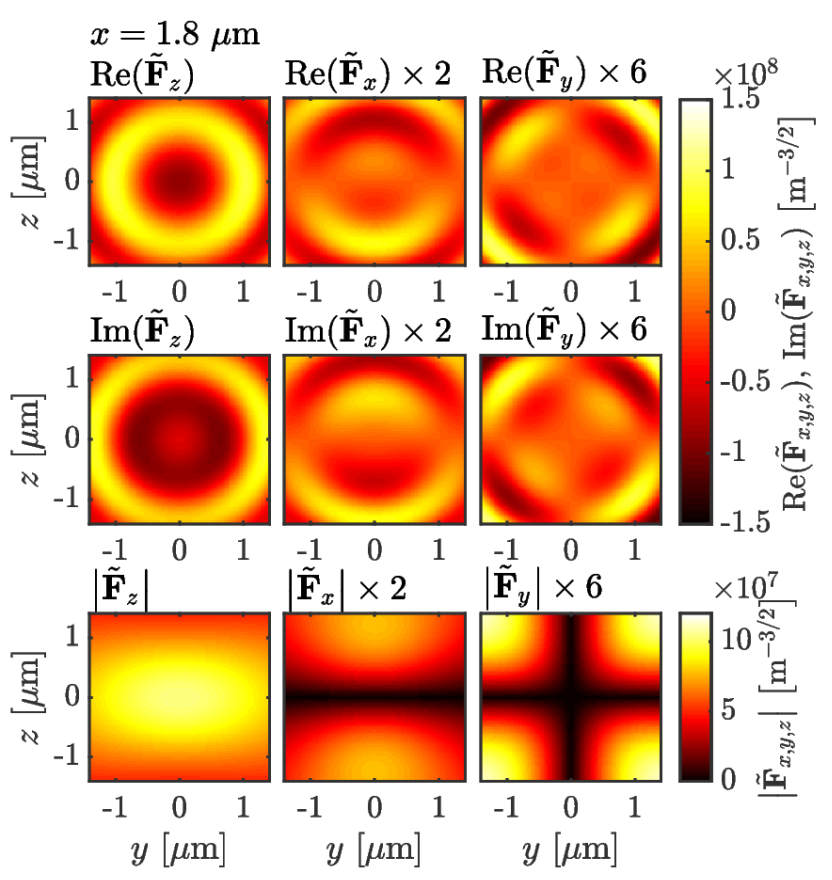

Figure 9. $\tilde{\mathbf{F}}_{z}, \tilde{\mathbf{F}}_{x}$ and $\tilde{\mathbf{F}}_{y}$ at plane $x=1800 \mathrm{~nm}$ for $\gamma_{\mathrm{p}}=\gamma_{\mathrm{p} 0}$ using NF2FF transformation (Eqs. (28)) from the near field surface $h=50 \mathrm{~nm}$. The ranges of $z$ and $x$ are $(-2 \lambda, 2 \lambda)$, where $\lambda \approx 700 \mathrm{~nm}$. $z$-component is the dominant one. The $x$ and $y$ components are multiplied by a factor of 2 and 6 to better display the field distribution.

Moreover, as shown in Fig. $10(\mathrm{~b}), S^{\text {nrad }}$ and $S_{\mathrm{p}}^{\text {nrad }}$ increase with larger material losses, where only the results directly from COMSOL are presented. Meanwhile, $\beta_{\mathrm{QNM}}^{\text {nrad }}$ (Eq. (12)) from single QNM, and $\beta_{\text {num }}^{\text {nrad }}($ Eq. (81)) from the full dipole method are also shown in Fig. 10 (b), which are very close to each other, indicating that single mode approximation is an excellent one for these resonators (and also confirms the accuracy of our numerical calculations). Furthermore, these two classical nonradiative beta factors are very close to the quantum $S^{\text {nrad }}$ and $S_{\mathrm{p}}^{\text {nrad }}$.

The spatial integration contribution to $S^{\text {nrad }}$, is defined through:

$$
\left.S^{\mathrm{nrad}}\right|_{\text {space }}=\int_{V} d \mathbf{r}\left|\tilde{\mathbf{f}}_{\mathrm{c}}(\mathbf{r})\right|^{2}
$$

Table II. Spatial integration $\left.S^{\text {nrad }}\right|_{\text {space }}$ (Eq. (82)), quoted to the third decimal place

\begin{tabular}{|c|c|c|c|c|c|}
\hline spatial integral & $3 \gamma_{\mathrm{p} 0}$ & $2 \gamma_{\mathrm{p} 0}$ & $\gamma_{\mathrm{p} 0}$ & $(2 / 3) \gamma_{\mathrm{p} 0}$ & $(1 / 3) \gamma_{\mathrm{p} 0}$ \\
\hline grid $1 \mathrm{~nm}$ & 0.059 & 0.059 & 0.059 & 0.059 & 0.059 \\
\hline grid $0.5 \mathrm{~nm}$ & 0.045 & 0.045 & 0.045 & 0.045 & 0.045 \\
\hline grid $0.2 \mathrm{~nm}$ & 0.041 & 0.041 & 0.041 & 0.041 & 0.041 \\
\hline grid $0.1 \mathrm{~nm}$ & 0.040 & 0.040 & 0.040 & 0.040 & 0.040 \\
\hline COMSOL & 0.039 & 0.039 & 0.039 & 0.039 & 0.039 \\
\hline
\end{tabular}
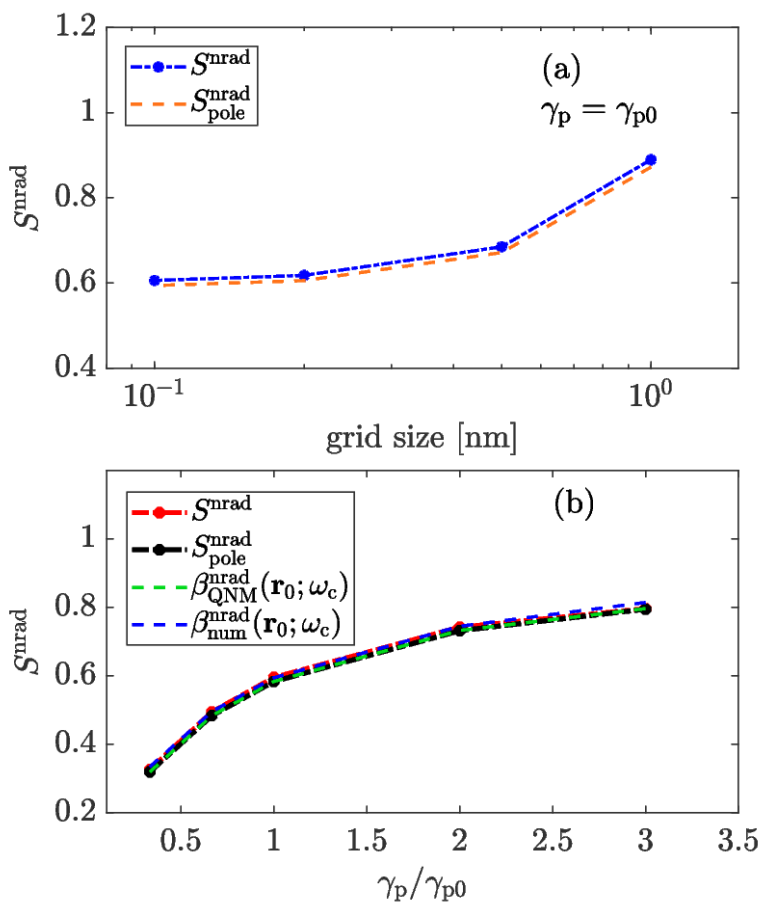

Figure 10. (a) Numerical grid dependence of $S^{\text {nrad }}$ (Eq. (51)) and $S_{\mathrm{p}}^{\text {nrad }}$ (Eq. (69)) for $\gamma_{\mathrm{p}}=\gamma_{\mathrm{p} 0}$. If the spatial volume integration is directly performed in COMSOL (i.e., no need to artificially choose the grid), the results are $S^{\text {nrad }}=0.5947$ and $S_{\mathrm{p}}^{\text {nrad }}=0.5830$, which is very close to the results with grid size of $0.1 \mathrm{~nm}$. (b) $S^{\text {nrad }}$ (Eq. (51)), $S_{\mathrm{p}}^{\text {nrad }}$ (Eq. (69)), $\beta_{\mathrm{QNM}}^{\text {nrad }}$ (Eq. (12)) from single QNM, and $\beta_{\text {num }}^{\text {nrad }}($ Eq. (81)) from full dipole method for various losses, where only the results directly from COMSOL are presented. Once gaain, the full dipole simulations are performed at gap center.

which was found to be very similar for all the five cases studies, as shown in Table II. Thus, according to Eq. (69), the corresponding $S_{\mathrm{p}}^{\text {nrad }}$ will be proportional to $Q \epsilon_{\mathrm{I}}\left(\omega_{\mathrm{c}}\right)$. If $\gamma_{\mathrm{p}}(0.0928 \mathrm{eV})$ is much smaller than $\omega_{\mathrm{c}}$ $(1.7798 \mathrm{eV})$, then

$$
\epsilon_{\mathrm{I}}\left(\omega_{\mathrm{c}}\right)=\frac{\omega_{\mathrm{p}}^{2} \gamma_{\mathrm{p}}}{\omega_{\mathrm{c}}^{3}+\omega_{\mathrm{c}} \gamma_{\mathrm{p}}^{2}} \approx \frac{\omega_{\mathrm{p}}^{2} \gamma_{\mathrm{p}}}{\omega_{\mathrm{c}}^{3}}
$$

Since $\omega_{\mathrm{c}}$ for these five cases are also very close, we expect that $S_{\mathrm{p}}^{\text {nrad }}$ will be proportional to $Q \gamma_{\mathrm{p}}$. We calculate $S_{\mathrm{p}}^{\text {nrad }}=0.5830$ for $\gamma_{\mathrm{p}}=\gamma_{\mathrm{p} 0}$, and can estimate $S_{\mathrm{p}}^{\text {nrad }} \approx 0.805,0.736,0.483,0.319$ for the other four cases, which are very close to the full computed values $S_{\mathrm{p}}^{\text {nrad }}$ $(0.790,0.731,0.483,0.319)$ shown in Fig. 10 (b); as expected, more accurate agreements with this simpler scaling argument is obtained for smaller $\gamma_{\mathrm{p}}$ i.e., larger $Q_{\mathrm{c}}$. 


\section{Calculation of $S^{\mathrm{rad}}$ using the regularized QNMs}

$$
\text { 1. Pole approximation } S_{\mathrm{p} 1}^{\mathrm{rad}}(E q .(70))
$$

Next we use the NF2FF approach to calculate $S^{\mathrm{rad}}$, which is a much more involved numerical calculation. We begin by considering on the first pole result $S_{\mathrm{p} 1}^{\mathrm{rad}}(\mathrm{Eq}$. (70)). The grid size in the near field surface $h$ is set as $0.5 \mathrm{~nm}$; the far field surface is fixed at $h_{\mathrm{far}}=630 \mathrm{~nm}$, and the grid on that surface is $20 \mathrm{~nm}$ (in both transverse directions). The near field surface dependence of pole $S_{\mathrm{p} 1}^{\mathrm{rad}}$ for various material loss cases are shown in Table III. $S_{\mathrm{p} 1}^{\mathrm{rad}}$ from $h=20 \mathrm{~nm}, h=30 \mathrm{~nm}$, and $h=50$ $\mathrm{nm}$ are very close, which appears to be more robust as expected. Deviations then start to occur for distances greater than $70 \mathrm{~nm}$ or so, as the QNM is no longer a good approximation to use for the near field currents. In principle, the near field surface should be as close as possible to the metal surface. However, numerically, the fields close to the metal surface have a large gradient (and convergence problems at the metal surface), so it will (at least) need smaller grid size (smaller than $0.5 \mathrm{~nm}$ now used) at near field surfaces to guarantee the accuracy of numerical results. So in following calculations, we mainly choose $h=50 \mathrm{~nm}$ as near field surface. The run time is around 16 minutes.

Table III. Near field surface dependence of $S_{\mathrm{p} 1}^{\mathrm{rad}}$ (Eq. (70)), quoted to the second decimal place for various material loss cases.

\begin{tabular}{|c|c|c|c|c|c|}
\hline NF surface & $3 \gamma_{\mathrm{p} 0}$ & $2 \gamma_{\mathrm{p} 0}$ & $\gamma_{\mathrm{p} 0}$ & $(2 / 3) \gamma_{\mathrm{p} 0}$ & $(1 / 3) \gamma_{\mathrm{p} 0}$ \\
\hline$h=10 \mathrm{~nm}$ & 0.19 & 0.26 & 0.41 & 0.51 & 0.68 \\
\hline$h=20 \mathrm{~nm}$ & 0.19 & 0.26 & 0.42 & 0.52 & 0.68 \\
\hline$h=30 \mathrm{~nm}$ & 0.19 & 0.26 & 0.42 & 0.52 & 0.68 \\
\hline$h=50 \mathrm{~nm}$ & 0.20 & 0.27 & 0.42 & 0.52 & 0.69 \\
\hline$h=70 \mathrm{~nm}$ & 0.21 & 0.28 & 0.44 & 0.54 & 0.70 \\
\hline
\end{tabular}

Table IV. Influence of far field location on the pole calculation for $S_{\mathrm{p} 1}^{\mathrm{rad}}($ Eq. $(70))$, using $\gamma_{\mathrm{p}}=\gamma_{\mathrm{p} 0}$. Here the near field surface is fixed at $h=50 \mathrm{~nm}$ and resonance wavelength is about $\lambda \sim 700 \mathrm{~nm}(1.78 \mathrm{eV})$. When the propagation distance $h_{\mathrm{far}}-h$ is larger than $0.5 \lambda, S_{\mathrm{p} 1}^{\mathrm{rad}}$ is well converged. The values are quoted to the second decimal place

$$
\begin{array}{|l|l|}
\hline h_{\text {far }}=410 \mathrm{~nm} & 0.42 \\
\hline h_{\text {far }}=590 \mathrm{~nm} & 0.42 \\
\hline h_{\text {far }}=630 \mathrm{~nm} & 0.42 \\
\hline h_{\text {far }}=750 \mathrm{~nm} & 0.42 \\
\hline h_{\text {far }}=990 \mathrm{~nm} & 0.42 \\
\hline
\end{array}
$$

In Table IV, we summarize the impact of the far field surface $h_{\text {far }}$ selection on the $S_{\mathrm{p} 1}^{\mathrm{rad}}$ (Eq. (70)), with the near field surface fixed at $h=50 \mathrm{~nm}$. The resonance wavelength here is about $\lambda \sim 700 \mathrm{~nm}(1.78 \mathrm{eV})$. With $\gamma_{\mathrm{p}}=\gamma_{\mathrm{p} 0}$, choosing far field surfaces at $h_{\mathrm{far}}=$ $410,590,630,750,990 \mathrm{~nm}$ gave the same pole result of $S_{\mathrm{p} 1}^{\mathrm{rad}}=0.42$.
2. The second pole approximation $S_{\mathrm{p} 2}^{\mathrm{rad}}(E q .(71))$ with field equivalence

Next we considerthe second pole result $S_{\mathrm{p} 2}^{\mathrm{rad}}$ from Eq. (71). There are several influencing factors when performing the numerical integrals in $I_{c}$ (Eq. (72)), including the selection of near field surface $\left(S^{\prime}\right)$ and angle grid size for angle integral. Here we fix the grid size in near field surface with a spacing of $0.5 \mathrm{~nm}$, and we use the same angle grid for integration over both $\vartheta$ and $\varphi$.

Table V. Pole calculation for $S_{\mathrm{p} 2}^{\mathrm{rad}}$ from Eq. (71) with $h=50$ $\mathrm{nm}$ and $\gamma_{\mathrm{p}}=\gamma_{\mathrm{p} 0}$, quoted to the second decimal place.

\begin{tabular}{|c|c|c|}
\hline near field surface $h=50 \mathrm{~nm}$ & & \\
\hline angle grid for $\vartheta$ and $\varphi$ & run time & pole $S_{\mathrm{p} 2}^{\mathrm{rad}}$ \\
\hline$\pi / 2$ & 3.2 secs & 0.51 \\
\hline$\pi / 3$ & 4.8 secs & 0.42 \\
\hline$\pi / 5$ & 8.7 secs & 0.42 \\
\hline$\pi / 10$ & 25.3 secs & 0.42 \\
\hline \multicolumn{2}{|r|}{} \\
\hline
\end{tabular}

Table VI. Pole calculation for $S_{\mathrm{p} 2}^{\mathrm{rad}}$ from Eq. (71) with $h=30$ $\mathrm{nm}$ and $\gamma_{\mathrm{p}}=\gamma_{\mathrm{p} 0}$, quoted to the second decimal place.

\begin{tabular}{|c|c|c|}
\hline near field surface $h=30 \mathrm{~nm}$ & & \\
\hline angle grid for $\vartheta$ and $\varphi$ & run time & pole $S_{\mathrm{p} 2}^{\mathrm{rad}}$ \\
\hline$\pi / 2$ & 1.9 secs & 0.51 \\
\hline$\pi / 3$ & 2.8 secs & 0.42 \\
\hline$\pi / 5$ & 4.9 secs & 0.42 \\
\hline$\pi / 10$ & 13.9 secs & 0.42 \\
\hline
\end{tabular}

The main numerical results for obtaining the pole calculation $S_{\mathrm{p} 2}^{\mathrm{rad}}$ (Eq. (71)) are shown in Tables V and VI, showing a convergent solutions of 0.42 (quoted to the second decimal place) with near field surface $h=50 \mathrm{~nm}$ and $30 \mathrm{~nm}$ for $\gamma_{\mathrm{p}}=\gamma_{\mathrm{p} 0}$. These also agree with the first pole result $S_{\mathrm{p} 1}^{\mathrm{rad}}$ of Eq. (70) (Table III), but with a significantly faster run time (around $100-200$ times faster).

Moreover, the results of $S_{\mathrm{p} 2}^{\mathrm{rad}}$ (Eq. (71)) for various material losses and with fixed near field surface $h=50$ $\mathrm{nm}$ (grid size is $0.5 \mathrm{~nm}$ ) are shown in Table VII, which increase with the decrease of the loss, and they are very close to the corresponding $S_{\mathrm{p} 1}^{\mathrm{rad}}$ (Eq. (70)). We have also found that pole $S_{\mathrm{p} 2}^{\mathrm{rad}}$ always converges at a relatively large angle grid size, and thus the calculation is extremely fast, only a few seconds to several tens of seconds.

3. Computing $S^{\mathrm{rad}}(E q .(52))$ with a numerical frequency
integration versus the two pole approximations $S_{\mathrm{p} 1}^{\mathrm{rad}}$ (Eq. (70)) and $S_{\mathrm{p} 2}^{\mathrm{rad}}(E q .(71))$

Next, we carry out the full frequency integration results $S^{\text {rad }}$ (Eq. (52)), again using the NF2FF transformation. Figure 11 (a) show the normalized function $\left|A_{\mathrm{c}}(\omega)\right|^{2}$ versus frequency. The black, red, and green perpendicular lines indicate the frequency position at $\omega_{\mathrm{c}}, \omega_{\mathrm{c}}+12 \gamma_{\mathrm{c}}$, 
$\omega_{\mathrm{c}}+14 \gamma_{\mathrm{c}}$. The integrated frequency region $\left(0, \omega_{\mathrm{c}}+12 \gamma_{\mathrm{c}}\right)$ and $\left(0, \omega_{c}+14 \gamma_{c}\right)$ cover the vast majority of the QNM lineshape, as discussed earlier. Figure 11 (b) show the full frequency integration results vs the pole results with $\gamma_{\mathrm{p}}=\gamma_{\mathrm{p} 0}, h=50 \mathrm{~nm}$ and $h_{\mathrm{far}}=630 \mathrm{~nm}$.
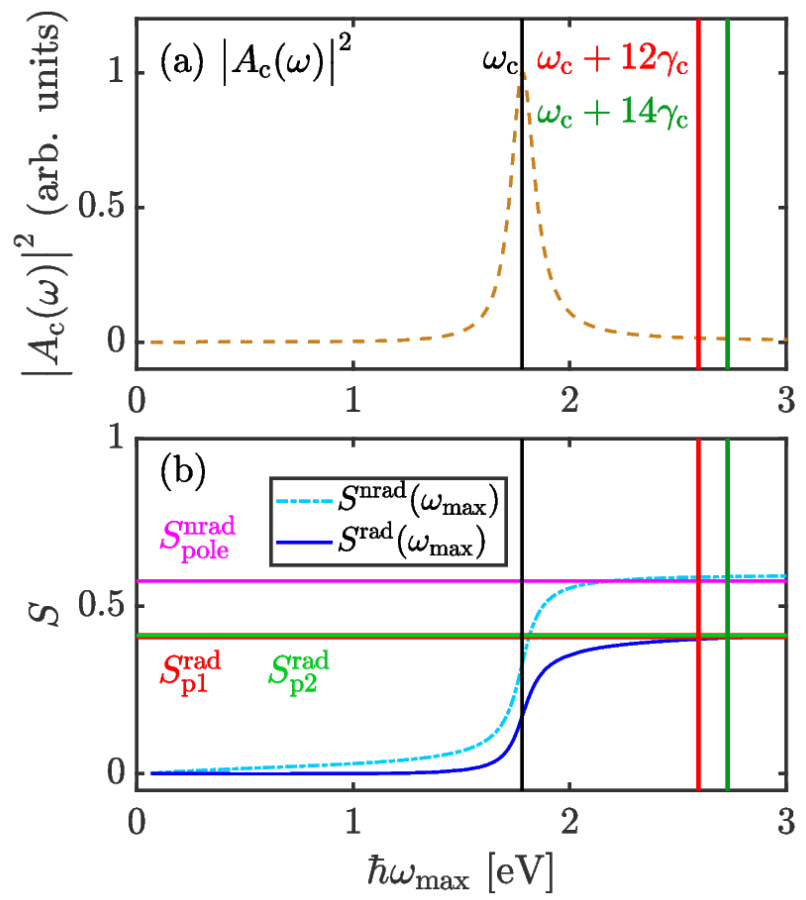

Figure 11. (a) Plot of $\left|A_{\mathrm{c}}(\omega)\right|^{2}$ versus frequency (normalized to 1). The black, red, and green perpendicular lines indicate the frequency position at $\omega_{\mathrm{c}}, \omega_{\mathrm{c}}+12 \gamma_{\mathrm{c}}, \omega_{\mathrm{c}}+14 \gamma_{\mathrm{c}}$. (b) Full frequency integral $S^{\text {nrad }}$ (Eq. (51)), pole result $S_{\mathrm{p}}^{\text {nrad }}$ (Eq. (69)), full frequency result $S^{\text {rad }}$ (Eq. (52)), pole result $S_{\mathrm{p} 1}^{\mathrm{rad}}$ (Eq. (70)) and pole results $S_{\mathrm{p} 2}^{\mathrm{rad}}($ Eq. (71)) for $h=50 \mathrm{~nm}$ and $h_{\mathrm{far}}=630 \mathrm{~nm}$ with $\gamma_{\mathrm{p}}=\gamma_{\mathrm{p} 0}$. In detail, $S_{\mathrm{p}}^{\mathrm{nrad}}=0.583$. $S^{\mathrm{nrad}}\left(\omega_{\max }=\omega_{\mathrm{c}}+12 \gamma_{\mathrm{c}}\right)=0.587 . S^{\mathrm{nrad}}\left(\omega_{\max }=\omega_{\mathrm{c}}+14 \gamma_{\mathrm{c}}\right)=$ 0.589. $S_{\mathrm{p} 1}^{\mathrm{rad}}=0.423 . S_{\mathrm{p} 2}^{\mathrm{rad}}=0.418 . S^{\mathrm{rad}}\left(\omega_{\max }=\omega_{\mathrm{c}}+12 \gamma_{\mathrm{c}}\right)=$ 0.402. $S^{\mathrm{rad}}\left(\omega_{\max }=\omega_{\mathrm{c}}+14 \gamma_{\mathrm{c}}\right)=0.406$.

With regards to the pole results, for the nonraditive contribution, $S_{\mathrm{p}}^{\text {nrad }}=0.583$ (Eq. (51)) (magenta horizontal line). Cyan line show $S^{\text {nrad }}$ as a function of the maximum integral frequency; integrating over frequency, then we obtain $S^{\text {nrad }}\left(\omega_{\max }=\omega_{\mathrm{c}}+12 \gamma_{\mathrm{c}}\right)=0.587$. $S^{\text {nrad }}\left(\omega_{\max }=\omega_{\mathrm{c}}+14 \gamma_{\mathrm{c}}\right)=0.589$. These values are very close to the pole result, which confirms the accuracy of the pole approximation $S_{\mathrm{p}}^{\text {nrad }}$. For the radiative contribution, $S_{\mathrm{p} 1}^{\mathrm{rad}}=0.423$ (Eq. (70)) (red horizontal line), $S_{\mathrm{p} 2}^{\mathrm{rad}}=0.418$ (Eq. (71)) (green horizontal line), while the blue curve shows $S^{\mathrm{rad}}$ (Eq. (52)) as a function of the maximum integral frequency ( 56 frequency points are used). These results confirm the accuracy of both two pole approximations $S_{\mathrm{p} 1}^{\mathrm{rad}}$ and $S_{\mathrm{p} 2}^{\mathrm{rad}}$, though the latter is considerably more efficient.

\section{Summary of quantum $S$ parameter for metal dimers}

Here we summarize the quantum pole $S$ parameters, $S_{\mathrm{p}}^{\text {nrad }}\left(\right.$ Eq. (69)) and $S_{\mathrm{p}}^{\mathrm{rad}}=S_{\mathrm{p} 2}^{\mathrm{rad}}$ (Eq. $\left.(71)\right)$, for gold dimer with different materials loss $\gamma_{\mathrm{p}}$ in Table VII. As the material loss $\gamma_{\mathrm{p}}$ decreases $\left(Q_{\mathrm{c}}\right.$ increases $), S_{\mathrm{p}}^{\text {nrad }}$ decreases and $S_{\mathrm{p}}^{\mathrm{rad}}$ increases. Somewhat remarkably though, the total $S_{\mathrm{p}}=S_{\mathrm{p}}^{\mathrm{nrad}}+S_{\mathrm{p}}^{\mathrm{rad}}$ for these five cases are all close to 1.0. While this may be expected for a single QNM, a general proof is not known, and the complexity of the numerical calculations also confirm the general accuracy of the numerical implementation.

For completeness, we have also listed the quantum radiative beta factor $\beta_{\text {quan }}^{\mathrm{rad}}=S_{\mathrm{p}}^{\mathrm{rad}} / S_{\mathrm{p}}$ (Eq. (57)), classical $\beta_{\mathrm{QNM}}^{\mathrm{rad}}=\beta_{\mathrm{QNM}}^{\mathrm{rad}}\left(\mathbf{r}_{0}, \omega_{\mathrm{c}}\right)(\mathrm{Eq}$. (12)) with single QNM, and classical $\beta_{\text {num }}^{\text {rad }}=\beta_{\text {num }}^{\text {rad }}\left(\mathbf{r}_{0}, \omega_{\mathrm{c}}\right)$ (Eq. (80)) with full dipole method in Table VII. The last two classical beta factor are evaluated at the pole frequency $\omega_{c}$ for a emitter placed at dimer center $\mathbf{r}_{0}$. We found that the quantum and classical radiative beta factor are relatively close to each other and they increase with $Q_{\mathrm{c}}$ increase. However, note that the classical estimation (with full dipole method) is really a total beta calculation as opposed to a single mode, but they are likely very close in this regime.

Table VII. Pole result for $S_{\mathrm{p}}^{\mathrm{nrad}}$ (Eq. (69)) and $S_{\mathrm{p}}^{\mathrm{rad}}=S_{\mathrm{p} 2}^{\mathrm{rad}}$ (Eq. (71)) with different materials loss $\gamma_{\mathrm{p}}$. The total $S_{\mathrm{p}}=$ $S_{\mathrm{p}}^{\text {nrad }}+S_{\mathrm{p}}^{\mathrm{rad}}$. The quantum radiative beta factor $\beta_{\mathrm{quan}}^{\mathrm{rad}}=$ $S_{\mathrm{p}}^{\mathrm{rad}} / S_{\mathrm{p}}$. Here, classical beta factor $\beta_{\mathrm{QNM}}^{\mathrm{rad}}=\beta_{\mathrm{QNM}}^{\mathrm{rad}}\left(\mathbf{r}_{0}, \omega_{\mathrm{c}}\right)$ (Eq. (12)) and $\beta_{\text {num }}^{\text {rad }}=\beta_{\text {num }}^{\text {rad }}\left(\mathbf{r}_{0}, \omega_{\text {c }}\right)($ Eq. $(80))$, which are evaluated at the pole frequency $\omega_{\mathrm{c}}$ for a emitter placed at dimer center $\mathbf{r}_{0}$. All $S$ and beta factors are quoted to the second decimal place.

\begin{tabular}{|c|c|c|c|c|c|c|c|}
\hline$\gamma_{\mathrm{p}}$ & $Q_{\mathrm{c}}$ & $S_{\mathrm{p}}^{\mathrm{nrad}}$ & $S_{\mathrm{p}}^{\mathrm{rad}}$ & $S_{\mathrm{p}}$ & $\beta_{\mathrm{quan}}^{\mathrm{rad}}$ & $\beta_{\mathrm{QNM}}^{\mathrm{rad}}$ & $\beta_{\text {num }}^{\mathrm{rad}}$ \\
\hline $3 \gamma_{\mathrm{p} 0}$ & 6.0 & 0.79 & 0.19 & 0.99 & 0.20 & 0.20 & 0.19 \\
\hline $2 \gamma_{\mathrm{p} 0}$ & 8.3 & 0.73 & 0.26 & 1.00 & 0.27 & 0.27 & 0.26 \\
\hline$\gamma_{\mathrm{p} 0}$ & 13.1 & 0.58 & 0.42 & 1.00 & 0.42 & 0.42 & 0.41 \\
\hline$\frac{2}{3} \gamma_{\mathrm{p} 0}$ & 16.3 & 0.48 & 0.52 & 1.00 & 0.52 & 0.52 & 0.50 \\
\hline$\frac{1}{3} \gamma_{\mathrm{p} 0}$ & 21.5 & 0.32 & 0.68 & 1.00 & 0.68 & 0.68 & 0.67 \\
\hline
\end{tabular}

\section{Quantum Purcell factor for the metal gold dimer}

For completeness, we now show the quantum Purcell factor in the bad cavity limit, using Eq. (56). Considering again a $z$-polarized dipole placed at gold cylindrical dimer gap center with $\gamma_{\mathrm{p}}=\gamma_{\mathrm{p} 0}$, then Fig. 12 shows the excellent agreement with the full dipole formula Eq. (79) (Fig. 12). Here, as shown in in Table VII, the calculated pole $S_{\mathrm{p}}=$ 1.00 is used. 


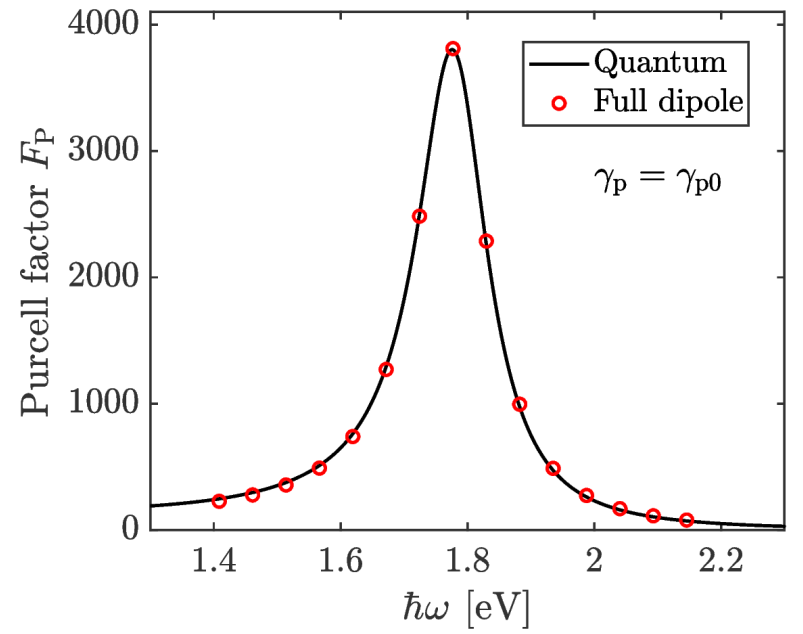

Figure 12. Quantum Purcell factor calculations for a $z$ polarized dipole at dimer gap center, with $\gamma_{\mathrm{p}}=\gamma_{\mathrm{p} 0}$ (gold), using the quantum formula (Eq. (56)), where pole $S_{\mathrm{p}}=1.0$ (shown in Table VII). The quantum Purcell factors (black curve) show excellent agreement with the result from full dipole formula (red circle, Eq. (79)).

\section{E. Run time comparison between the Dyson} approach and the NF2FF approach to compute the quantum $S$ parameters

Before showing the run times, we briefly clarify the simplification used to calculate $S^{\text {rad }}$ (Eq. (52)) and the pole result for $S_{\mathrm{p} 1}^{\mathrm{rad}}$ (Eq. (70)). We need to perform an integral involving $\tilde{\mathbf{F}}$ and $\tilde{\mathbf{H}}$ over a far field surface $\left(h_{\text {far }}\right)$, and we also know that the radiation of the QNM we are working with is axial-symmetric to the long axis of the $\mathrm{Au}$ dimer, and is also symmetrical to the center plane perpendicular to the long axis. Thus we can simplify this surface integral using symmetry (for this specific problem at least).

As shown in Fig. 6, we select a cylindrical surface as the far field surface. The surface integral unit is labeled as dS. If we select the grid size of $20 \mathrm{~nm}$, then $\mathrm{dS}=(20$ $\mathrm{nm})^{2}$. On the one hand, because it is symmetrical to the center plane, we can just calculate the integral over upper part of the cylindrical surfacel, and simply multiply the result by the factor of 2 . On the other hand, since it is axial-symmetric, we only need to perform the integral over a line (both in side surface and top surface), and then multiply the results by the length of the circumference (it is actually circumference/grid size). Note that this simplification may not work for other resonator structures if they do not have a similar symmetry, such as the coupled QNM case shown in Section IV.

\section{Dyson equation run times for computing the quantum $S$ parameters}

Here we estimate typical computational run times to obtain $S^{\mathrm{rad}}$ (Eq. (52)), $S_{\mathrm{p} 1}^{\mathrm{rad}}$ (Eq. (70)) and $S_{\mathrm{p} 2}^{\mathrm{rad}}(\mathrm{Eq}$. (71)) at Table VIII. First, for the Dyson approach, we selected the grid size as $0.2(0.5) \mathrm{nm}$ for the spatial integration over the metal volume. As mentioned in Sec. II B, the run time for a single $\tilde{\mathbf{F}}$ point at single frequency is about $\approx 8.5-10(0.6-0.7)$ minutes. We choose the far field cylindrical surface at $h_{\mathrm{far}}=630 \mathrm{~nm}$, and the grid at this surface is $20 \mathrm{~nm}$. Exploiting the symmetry mentioned above, then 33 and 37 points (both $\tilde{\mathbf{F}}$ and $\tilde{\mathbf{H}}$ ) are needed for the top surface (same for the bottom surface) and (half) side surface. Also note that $\tilde{\mathbf{H}}$ need to be calculated from curl of $\tilde{\mathbf{F}}$. Then 167 and $187 \tilde{\mathbf{F}}$ points are needed for the top (same for down) surface and (half) side surface of the far field. Using the $S^{\text {rad }}$ (Eq. (52)) results with a numnerical frequency integration, we use 56 frequency points (this is a typical number, and 51 points used in Ref. 33.) Thus, with inside grid of $0.2(0.5) \mathrm{nm}$, it will take about $8.5 \times 354 \times 56$ minutes $\approx 117$ days $(0.6 \times 354 \times 56$ minutes $\approx 8.3$ days $)$ for $S^{\text {rad }}($ Eq. $(52))$, and $8.5 \times 354$ minutes $\approx 2.1$ days $(0.6 \times 354$ minutes $\approx 3.5$ hours) for the pole result $S_{\mathrm{p} 1}^{\mathrm{rad}}(\mathrm{Eq} .(70))$.

\section{Near-field to far-field run times for computing the quantum $S$ parameters}

Next, if we employ the NF2FF transformation, we show how the run time will be greatly reduced. For example, considering a grid size of $0.5 \mathrm{~nm}$ at the near field surface (3D grid), and, similar to the above Dyson approach, the far field surface is selected at $h_{\mathrm{far}}=630 \mathrm{~nm}$ with a grid size of $20 \mathrm{~nm}$; the averaged time per spatial point at a single frequency is about 0.224 minutes (also shown in Sec. IIIB) and 0.123 minutes from the near field surface $h=50 \mathrm{~nm}$ and $h=30 \mathrm{~nm}$. Also note that, from the NF2FF theory, $\tilde{\mathbf{H}}$ does not need to be calculated from the curl of $\tilde{\mathbf{F}}$; these two are obtained at the same time from the vector potential (Eq. (28) and Eq. (29)). Thus for $h=50 \mathrm{~nm}$, it will take about $0.224 \times 70 \times 56$ minutes $\approx 14.6$ hours for $S^{\text {rad }}$ (Eq. (52)), and $0.2244 \times 70$ minutes $\approx 15.7$ minutes for pole result $S_{\mathrm{p} 1}^{\text {rad }}($ Eq. $(70))$. Similarly, for $h=30 \mathrm{~nm}$, it will take about $0.123 \times 70 \times 56$ minutes $\approx 8$ hours for $S^{\text {rad }}$ (Eq. (52)), and $0.123 \times 70$ minutes $\approx 8.6$ minutes for pole result $S_{\mathrm{p} 1}^{\mathrm{rad}}(\mathrm{Eq} .(70))$.

The run times for the pole result $S_{\mathrm{p} 2}^{\mathrm{rad}}$ (Eq. (71)) comes from Table V and Table VI, using the smallest time it take to get the convergent value. Clearly this method is extremely efficient and full calculations are completed in a few seconds.

We summarize the above run times using the Dyson and NF2FF approaches in Table VIII. For $S^{\mathrm{rad}}$ (Eq. (52)) and $S_{\mathrm{p} 1}^{\mathrm{rad}}($ Eq. (70)), the run times with the Dyson equation using grid $0.2(0.5) \mathrm{nm}$ will be about 192 (13.5) times) and 348 (24.6) times longer than the NF2FF trans- 
Table VIII. Comparison of example run times for $S^{\mathrm{rad}}$ (Eq. (52)), $S_{\mathrm{p} 1}^{\mathrm{rad}}\left(\right.$ Eq. (70)), and $S_{\mathrm{p} 2}^{\mathrm{rad}}$ (Eq. (71)) between the Dyson approach and the NF2FF approach. For the Dyson approach, the grid size for volume integration inside metal is 0.2 or $0.5 \mathrm{~nm}$. For NF2FF approach, the grid size used for near field surface (here $h=30 \mathrm{~nm}$ and $h=50 \mathrm{~nm}$ are shown) integration is selected as $0.5 \mathrm{~nm}$. Far field surface for both approaches is at $h_{\mathrm{far}}=630 \mathrm{~nm}$, and the grid at this surface is $20 \mathrm{~nm}$.

\begin{tabular}{|l|l|l|}
\hline time & Dyson, grid=0.2 $\mathrm{nm}$ & $\mathrm{NF} 2 \mathrm{FF}, h=50 \mathrm{~nm}$ \\
\hline$S^{\mathrm{rad}}$ & 117 days & 14.6 hours \\
\hline$S_{\mathrm{p} 1}^{\mathrm{rad}}$ & 2.1 days & $15.7 \mathrm{mins}$ \\
\hline$S_{\mathrm{p} 2}^{\mathrm{rad}}$ & & 4.8 secs \\
\hline & Dyson, grid $=0.5 \mathrm{~nm}$ & $\mathrm{NF} 2 \mathrm{FF}, h=30 \mathrm{~nm}$ \\
\hline$S^{\mathrm{rad}}$ & 8.3 days & 8 hours \\
\hline$S_{\mathrm{p} 1}^{\mathrm{rad}}$ & 3.5 hours & 8.6 mins \\
\hline$S_{\mathrm{p} 2}^{\mathrm{rad}}$ & & 2.8 secs \\
\hline
\end{tabular}

formation with the near field surface $h=50 \mathrm{~nm}$ and $h=30 \mathrm{~nm}$. Moreover, the run time for $S_{\mathrm{p} 2}^{\mathrm{rad}}(\mathrm{Eq}$. (71)) is only several seconds, which is promising to use with more complicated geometries such as the example below with coupled plasmon PC modes.

\section{NUMERICAL RESULTS FOR COUPLED QUASINORMAL MODES AND HYBRID METAL-DIELECTRIC SYSTEMS}

In this section, we focus on a much more complex example, which uses coupled modes formed by a metaldielectric system, with peak Purcell factors in excess of 1 million. These systems can exhibit rich interference effects and exploit some of the advantages of both cavity parts. For example, the plasmonic structure posses extreme localized fields enhancement (small mode volumes), but with relative low quality factor due to metallic losses ${ }^{4,6,8}$. In contrast, a PC cavity generally has a very high quality factor, but with a smaller mode volume that is limited by diffraction. Combining these systems together will get a range of cavity mode properties and line shapes (including Fano-like lineshapes ${ }^{48,49}$ ), which offer new possibilities ${ }^{29,50-53}$ that can benefit from the high quality factor of the dielectric structure and the significant field enhancements of the plasmonic structure. The challenge for obtaining the regularized modes of such a system is that the simple symmetry of the dimer cannot be exploited, and the spatial size of the system region is much larger in the case for the PC-like mode.

The hybrid system we model is shown in Fig. 13, which uses a gold $(\mathrm{Au})$ ellipsoid dimer (with center width of $W_{\mathrm{e}}=10 \mathrm{~nm}$, center length of $L_{\mathrm{e}}=50 \mathrm{~nm}$ and gap of $h_{\text {gap }}=2 \mathrm{~nm}$ ), put above a silicon-nitride photonic crystal beam (index $n_{\mathrm{PC}}=2.04$ ), similar to the coupled mode structures used in Refs. 33, 52, and 53. Specifically, the width and height of the beam is $W_{\text {beam }}=376 \mathrm{~nm}$ and
$L_{\text {beam }}=200 \mathrm{~nm}$; the length of the finite beam is $8.5 \mu \mathrm{m}$. The nearest distance between the dimer surface and the beam surface is $h_{\mathrm{d}}=5 \mathrm{~nm}$. The background medium is free space with refractive index $n_{\mathrm{B}}=1.0$, and again we use a Drude model with the same parameters as above for gold.

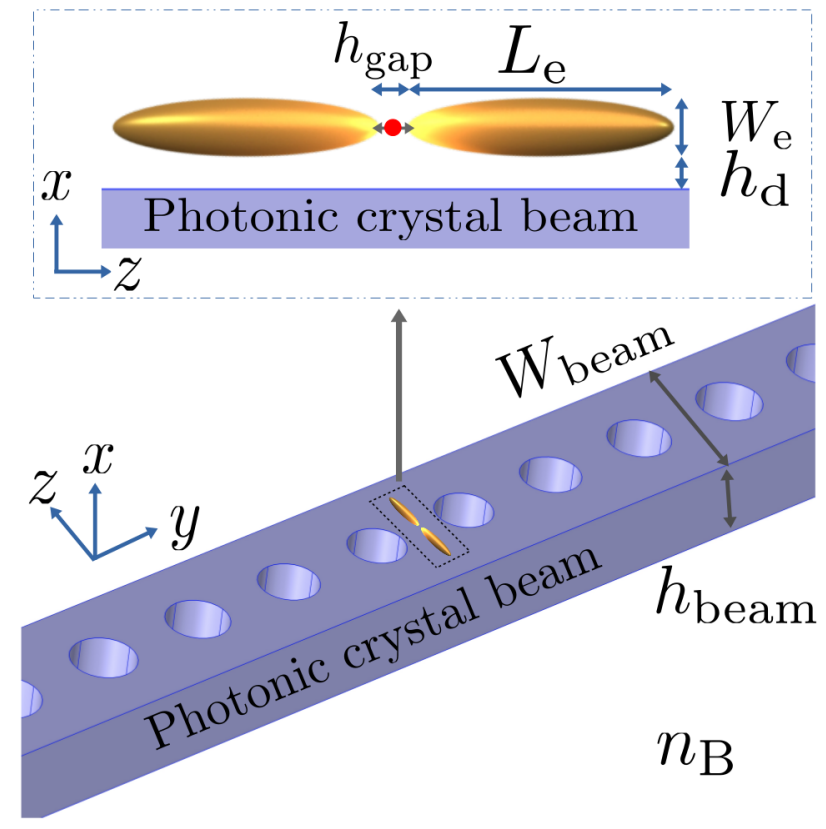

Figure 13. Schematic diagram of metal-dielectric hybrid structure, where a gold ellipsoid dimer is placed close to a PC cavity. A point dipole (red dot) polarized along $z$-direction is put at the gap center of the dimer. The origin of Cartesian coordinate system is located at dimer gap center. The length and width of the single ellipsoid are $L_{\mathrm{e}}=50 \mathrm{~nm}$ and $W_{\mathrm{e}}=10$ $\mathrm{nm}$. The gap of dimer is $h_{\text {gap }}=2 \mathrm{~nm}$, and the minimum distance between dimer surface and photonic crystal beam surface is $h_{\mathrm{d}}=5 \mathrm{~nm}$. The width and height of the beam are $W_{\text {beam }}=376 \mathrm{~nm}$ and $h_{\text {beam }}=200 \mathrm{~nm}$. The refractive index of beam is $n_{\mathrm{pc}}=2.04$, and the background medium is free space $\left(n_{\mathrm{B}}=1\right)$.

We use the same approach as shown in Sec. III A, to compute the QNMs: The scattered electric field of a point dipole at position $\mathbf{r}_{0}$ is related to the Green's function, and given by

$$
\mathbf{E}^{\mathrm{S}}(\mathbf{r}, \omega)=\frac{1}{\epsilon_{0}} \mathbf{G}\left(\mathbf{r}, \mathbf{r}_{0}, \omega\right) \cdot \mathbf{d},
$$

where $\mathbf{d}$ is the dipole moment of the emitter. If several modes are dominant in the regime of interest, then we can expand the Green's function with several QNMs,

$$
\mathbf{E}^{\mathrm{s}}(\mathbf{r}, \omega)=\sum_{\mu} \frac{1}{\epsilon_{0}} A(\omega) \tilde{\mathbf{f}}_{\mu}(\mathbf{r}) \tilde{\mathbf{f}}_{\mu}\left(\mathbf{r}_{0}\right) \cdot \mathbf{d} .
$$

We assume these modes are orthogonal with each other, so that

$$
\mathbf{E}_{\mu}^{\mathrm{s}}(\mathbf{r}, \omega)=\frac{1}{\epsilon_{0}} A(\omega) \tilde{\mathbf{f}}_{\mu}(\mathbf{r}) \tilde{\mathbf{f}}_{\mu}\left(\mathbf{r}_{0}\right) \cdot \mathbf{d}
$$


and $\mathbf{E}^{\mathrm{s}}(\mathbf{r}, \omega)=\sum_{\mu} \mathbf{E}_{\mu}^{\mathrm{s}}(\mathbf{r}, \omega)$. Subsequently, using a similar method shown in Sec. III A, we obtain the normalized QNMs field as

$$
\tilde{\mathbf{f}}_{\mu}(\mathbf{r})=\sqrt{\frac{2 \epsilon_{0}\left(\tilde{\omega}_{\mu}-\omega\right)}{\omega \mathbf{d} \cdot \mathbf{E}_{\mu}^{\mathrm{s}}\left(\mathbf{r}_{0}, \omega\right)}} \mathbf{E}_{\mu}^{\mathrm{s}}(\mathbf{r}, \omega),
$$

where $\omega=\left(1-10^{-5}\right) \times \tilde{\omega}_{\mu}$, very close to the pole frequency.

The simulation volume of the cylindrical module is about $85 \mu \mathrm{m}^{3}$ (including PMLs), where the maximum mesh sizes are $0.1 \mathrm{~nm}, 3 \mathrm{~nm}, 50 \mathrm{~nm}$ and $120 \mathrm{~nm}$ at the dipole point (center of the gap), inside the ellipsoid dimer, PC beam and elsewhere. The hybrid structure is significantly larger than gold dimer on its own, and we use 10 perfectly matched layers (PMLs) to minimize boundary reflections (the number of layers and the total thickness of PMLs should be adjusted according to the size of the inside simulation domain and the inside mesh settings to show the better performance).

Over a broad bandwidth of several $\mathrm{eV}$, there are two dominant modes of interest in this coupled structure. The first one we term 'QNM 1' is a plasmon-like mode, with resonance frequency $\tilde{\omega}_{1}=1.6999-0.0479 i$ $\mathrm{eV}$ and relatively low quality factor $Q_{1}=17.8$. The QNM field at the gap center of the ellipsoid dimer is $\tilde{\mathbf{f}}_{1 z}\left(\mathbf{r}_{0}\right)=1.800 \cdot 10^{12}-4.692 i \cdot 10^{10}\left[\mathrm{~m}^{-3 / 2}\right]$ (z-component). The corresponding effective mode volume is $V_{1}^{\text {eff }} / \lambda_{1}^{3}=$ $7.9575 \times 10^{-7}$, where $V_{1}^{\text {eff }}=1 / \operatorname{Re}\left[\tilde{\mathbf{f}}_{1 z}^{2}\left(\mathbf{r}_{0}\right)\right]$ and $\lambda_{1}=$ $2 \pi c / \operatorname{Re}\left(\tilde{\omega}_{1}\right)$. The second QNM, 'QNM 2', is a PC-like mode, with resonance frequency $\tilde{\omega}_{1}=1.6052-0.0007 i$ $\mathrm{eV}$ and a relatively high quality factor $Q_{2}=1139.3$. The QNM field at the center of the ellipsoid dimer gap is $\tilde{\mathbf{f}}_{2 z}\left(\mathbf{r}_{0}\right)=2.108 \cdot 10^{11}+9.623 i \cdot 10^{10}\left[\mathrm{~m}^{-3 / 2}\right]$; the effective mode volume is $V_{2}^{\text {eff }} / \lambda_{2}^{3}=6.1697 \times 10^{-5}$, where $V_{2}^{\text {eff }}=1 / \operatorname{Re}\left[\tilde{\mathbf{f}}_{2 z}^{2}\left(\mathbf{r}_{0}\right)\right]$ and $\lambda_{2}=2 \pi c / \operatorname{Re}\left(\tilde{\omega}_{2}\right)$. Note that these effective mode volumes can also be negative ${ }^{29}$.

To show the differences of the two QNMs more clearly, the QNM spatial fields and phase distribution are shown in Fig. 14, where the phase is defined by $\tilde{\mathbf{f}}_{1 z}=\left|\tilde{\mathbf{f}}_{1 z}\right| e^{i \phi_{1}}$ and $\tilde{\mathbf{f}}_{2 z}=\left|\tilde{\mathbf{f}}_{2 z}\right| e^{i \phi_{2}}$. At the PC beam center surface $\left(x=-W_{\mathrm{e}} / 2-h_{\mathrm{d}}-h_{\text {beam }} / 2\right)$, two QNMs show very different fields distributions (Fig. 14 (a) and (f)). While at dimer center surface $(x=0)$, the two modes show similar fields distribution (Fig. 14 (b) and (g)), except $\left|\tilde{\mathbf{f}}_{1 z}\right|$ is an order of magnitude larger than $\left|\tilde{\mathbf{f}}_{2 z}\right|$. Also note that the fields at dimer center surface $(x=0)$ are three (two) orders of magnitude larger than those at PC beam center surface for plasmon-like mode (PC-like mode), which means the two modes mainly live around the dimer region. In addition, as shown in Fig. 14 (c) and $(\mathrm{h})$, the QNM phases for the two modes are very different at the dimer center surface. At the dimer gap center point $(x=y=z=0 \mathrm{~nm}), \cos \left(2 \phi_{1}\right)=0.999$ and $\cos \left(2 \phi_{2}\right)=0.655$ for two modes. The phase difference between them will result in the Fano-like lineshape ${ }^{48}$ in total Purcell factors for a dipole placed at dimer gap center (Fig. 15). Figures 14 (d), (e), (i), and (j) show the
QNM fields distribution at the surface $y \underset{\sim}{=} 0 \mathrm{~nm}$. Close to dimer region, they look similar, except $\left|\tilde{\mathbf{f}}_{1 z}\right|$ is an order of magnitude larger than $\left|\tilde{\mathbf{f}}_{2 z}\right|$. Clearly, most of the QNM fields live in near dimer region, especially for plasmon-like mode.

We also stress that we are testing an extreme example here, as the gap size is only $2 \mathrm{~nm}$. To test the accuracy of a two QNM description, the generalized classical Purcell factors for a point dipole placing at the dimer gap center are shown in Fig. 15 (a). The results from QNMs theory (black curve) (Eq. (16)) show excellent qualitative agreement with full dipole calculation (red circles, Eq. (79)). This clearly indicates the validity of the QNMs results. The green and blue lines present their respective contribution to Purcell factors, with QNM 2 contributing negatively (in a certain frequency regime). Note also that, if we focus on dip region, the results from QNMs theory (black curve) net negative (below the black dashed line) (lowest point $-1.2 \times 10^{4}$ ). This is most probably caused by the onset of quasi-static contributions, whose contribution would naturally result in a net positive total Purcell factor; such contributions can be added into the theory using a quasi-static Green function theory ${ }^{28}$. If quasi-static contributions are considered, then the total Purcell factor from QNMs theory will be net-positive. For larger dimer gaps, the contribution of quasi-static modes is negligible ${ }^{29}$.

Next, we discuss the calculation of the quantized QNM parameters. First, it is quite simple to calculate $S_{\mu \eta}^{\text {nrad }}$ for coupled modes, since we only need the QNM fields inside the metal, which is the same level of difficulty as the dimer calculation on its own. Using Eq. (36), with a numerical frequency integral, we obtain $S_{11}^{\text {nrad }}=0.905$, $S_{22}^{\text {nrad }}=0.904, S_{12}^{\text {nrad }}=-0.0014-0.0975 i$, and $S_{21}^{\text {nrad }}=$ $S_{12}^{\text {nrad* }}$. Moreover, using Eq. (64), with an accurate pole approximation, we obtain $S_{\mathrm{p}, 11}^{\text {nrad }}=0.894, S_{\mathrm{p}, 22}^{\text {nrad }}=0.904$, $S_{\mathrm{p}, 12}^{\text {nrad }}=-0.0042-0.0967 i$, and $S_{\mathrm{p}, 21}^{\text {nrad }}=S_{\mathrm{p}, 12}^{\text {nrad* }}$. Note that for $S_{11}^{\text {nrad }}, S_{22}^{\text {nrad }}, S_{\mathrm{p}, 11}^{\text {nrad }}$, and $S_{\mathrm{p}, 22}^{\text {nrad }}$, the spatial integrals over metal volume are directly done in COMSOL; and for $S_{12}^{\mathrm{nrad}}$ and $S_{\mathrm{p}, 12}^{\mathrm{nrad}}$, the grid size is $0.1 \mathrm{~nm}$.

Numerically, the main challenge is to obtain the radiative part of the quantum $S$ factors, $S_{\mu \eta}^{\mathrm{rad}}$, which generally require the regularized QNM fields $(\tilde{\mathbf{F}}$ and $\tilde{\mathbf{H}})$ at a surface surrounding the entire hybrid structures. To compute $\tilde{\mathbf{F}}$ at a single spatial point with the Dyson Equation requires a spatial integration over the entire hybrid structure (PC beam and dimer). If the grid size $0.2(0.5) \mathrm{nm}$ is used, then it will take about 48 days (3.4 days) to get $\tilde{\mathbf{F}}$ at a single spatial point for a single frequency. The run time for Dyson equation is proportional to the integral volume if the same grid size is used. The integral volume for this structure, with just the PC beam and the ellipsoid dimer, is around 8122 times larger than that for gold cylindrical dimer. Then correspondingly, the run times will increase in the same way. Moreover, since the size of the hybrid structure is on the order of several micrometers, then the area of the outside surface is very large. For instance, the 
QNM 1: Plasmon-like

(a) $\left|\tilde{\mathbf{f}}_{1 z}\right|$ at $\mathrm{PC}$ center

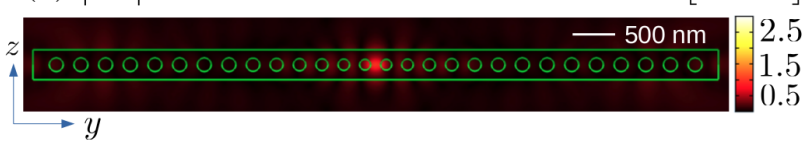

(b) $\left|\tilde{\mathbf{f}}_{1 z}\right|$

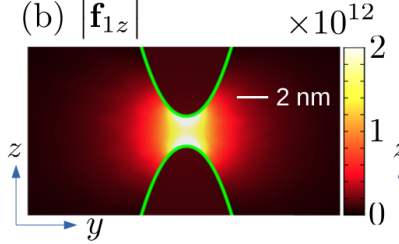

(c) $\cos \left(2 \phi_{1}\right)$

(d) $\left|\tilde{\mathbf{f}}_{1 z}\right| \quad \times 10^{11}$
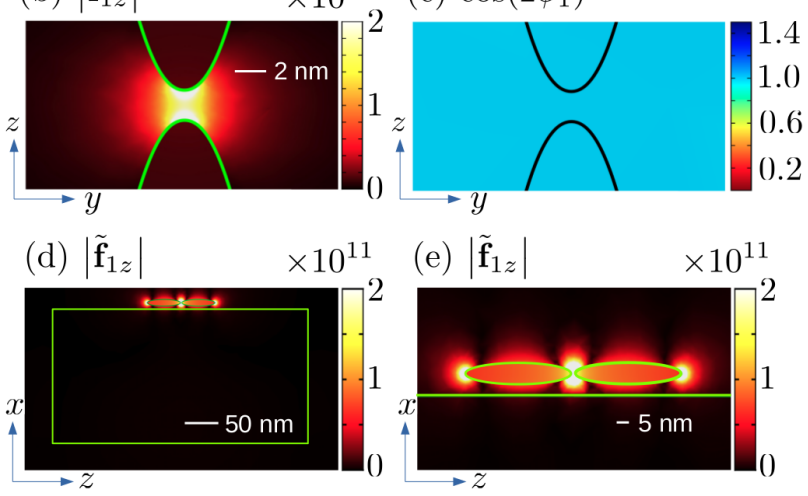

QNM 2: PC-like

(f) $\left|\tilde{\mathbf{f}}_{2 z}\right|$ at PC center

$\times 10^{9}\left[\mathrm{~m}^{-3 / 2}\right]$

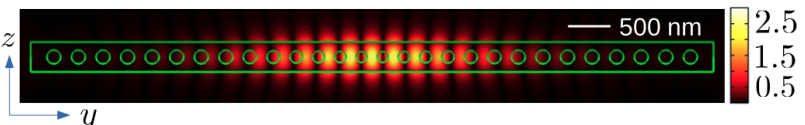

(g) $\left|\tilde{\mathbf{f}}_{2 z}\right|$

(h) $\cos \left(2 \phi_{2}\right)$
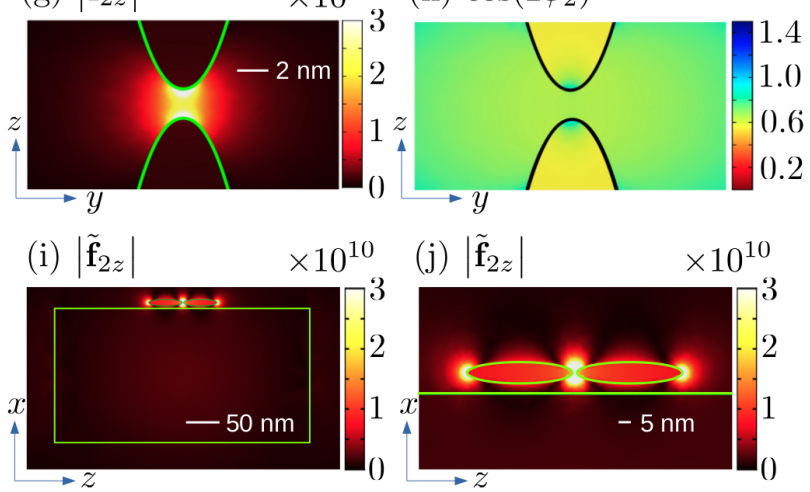

Figure 14. QNM spatial fields and phase distribution for the two QNMs of the hybrid metal-dielectric system. (a) $\left|\tilde{\mathbf{f}}_{1 z}\right|$ of QNM 1 (plasmon-like mode, low $Q$ ) at beam center surface $\left(x=-W_{\mathrm{e}} / 2-h_{\mathrm{d}}-h_{\text {beam }} / 2\right)$. Note that the origin of Cartesian coordinate system is located at dimer gap center point. (b) $\left|\tilde{\mathbf{f}}_{1 z}\right|$ of QNM 1 at surface $x=0$ nm (dimer center surface). (c) QNM phase $\cos \left(2 \phi_{1}\right)$ at $x=0 \mathrm{~nm}$, where phase is defined by $\tilde{\mathbf{f}}_{1 z}=\left|\tilde{\mathbf{f}}_{1 z}\right| e^{i \phi_{1}}$. At gap center point, $\cos \left(2 \phi_{1}\left(\mathbf{r}_{0}=[0,0,0]\right)\right)=0.999$. (d) $\left|\tilde{\mathbf{f}}_{1 z}\right|$ at $y=0 \mathrm{~nm}$. (e) Zoom in of (d). (f), (g), (h), (i), (j) Corresponding $\left|\tilde{\mathbf{f}}_{2 z}\right|$ and QNM phase $\cos \left(2 \phi_{2}\right)$ for QNM $2(\mathrm{PC}$-like mode, high $Q)$. At the gap center point, $\cos \left(2 \phi_{2}\left(\mathbf{r}_{0}=[0,0,0]\right)\right)=0.655$.

outside surface is chosen as a cuboid surface at $h_{\text {far }}=630$ $\mathrm{nm}$ (the smallest vertical distance to the dimer surface or PC beam surface); and the grid size at this surface is selected as $20 \mathrm{~nm}$. Although there is no similar symmetry as dimer only scenario, the fields are symmetric with respect to the $x-z$ and $x-y$ planes. Then we only need to do the integral over $1 / 4$ of this cuboid surface. Thus, in sumamry, it will take about 16114 (1141) years (!) with an inside grid size $0.2(0.5) \mathrm{nm}$ for even pole $S_{\mathrm{p} 1}^{\mathrm{rad}}$ (Eq. (70)) with Dyson equation. So clearly it is impractical to employ the Dyson equation to calculate $S_{\mu \eta}^{\mathrm{rad}}$ for such coupled modes.

To address this significant problem, we use the pole approximation (Eq. (67)) with a NF2FF transformation to calculate $S_{\mu \eta}^{\mathrm{rad}}$. For the plasmon-like mode, the near field surface is chosen as a cuboid surface just surrounding the dimer, since most of fields are located around the dimer (Fig. 14). The smallest vertical distance from the dimer to the five surfaces of this cuboid (not including the surface below) is $50 \mathrm{~nm}$; and in the $x$ - direction, the sixth surface is $4 \mathrm{~nm}$ below the lowest part of the dimer surface. For the PC-like mode, the near field surface is set as a cuboid surface surrounding the entire coupled structures, where the smallest vertical distance from the hybrid structure to every surface of this cuboid is $50 \mathrm{~nm}$ $(h=50 \mathrm{~nm})$. Note that, as shown in Appendix A, for the PC-like mode, the results from near field surface just surrounding dimer alone yield a very good approximation as well, since one can see that most of the PC-like mode fields are also located in the dimer region (see Fig. 14).
The grid sizes at these near field surfaces are $0.5 \mathrm{~nm}$ (the same as that for dimer only). The convergence tests for angle resolutions are shown in Table IX, X and XI. We got $S_{\mathrm{p} 2,11}^{\mathrm{rad}}=0.068, S_{\mathrm{p} 2,22}^{\mathrm{rad}}=0.134, S_{\mathrm{p} 2,12}^{\mathrm{rad}}=$ $-0.0021-0.0024 i$, and $S_{\mathrm{p} 2,21}^{\mathrm{rad}}=S_{\mathrm{p} 2,12}^{\mathrm{rad} *}$. As also shown in these table, the run times are several seconds to 1.7 hours, which is acceptable and significantly faster than those with Dyson approach (which are untractable for this geometry).

In summary, for the total $S$ values of the hybrid modes: $S_{\mathrm{p}, 11}=0.894+0.068, S_{\mathrm{p}, 22}=0.904+0.134, S_{\mathrm{p}, 12}=$ $(-0.0042-0.0967 i)+(-0.0021-0.0024 i), S_{\mathrm{p}, 21}=S_{\mathrm{p}, 12}^{*}$, where the first is nonradiative part and the second is radiative part. Then quantum Purcell factors (Eq. (47)) for coupled structures are shown in Fig. 15(c) and (d)

Table IX. Pole $S_{\mathrm{p} 2,11}^{\mathrm{rad}}$ from Eq. (67), quoted to the third decimal place. For the calculations, the plasmon-like mode uses a cuboid surface (with $h=50 \mathrm{~nm}$ ) that surrounds the dimer.

\begin{tabular}{|c|c|c|}
\hline near field surface $h=50 \mathrm{~nm}$ & & \\
\hline angle grid for $\vartheta$ and $\varphi$ & run time & pole $S_{\mathrm{p} 2,11}^{\mathrm{rad}}$ \\
\hline$\pi / 2$ & 2.0 secs & 0.081 \\
\hline$\pi / 3$ & 2.8 secs & 0.069 \\
\hline$\pi / 5$ & 5.0 secs & 0.068 \\
\hline$\pi / 8$ & 9.4 secs & 0.068 \\
\hline$\pi / 10$ & 12.4 secs & 0.068 \\
\hline
\end{tabular}



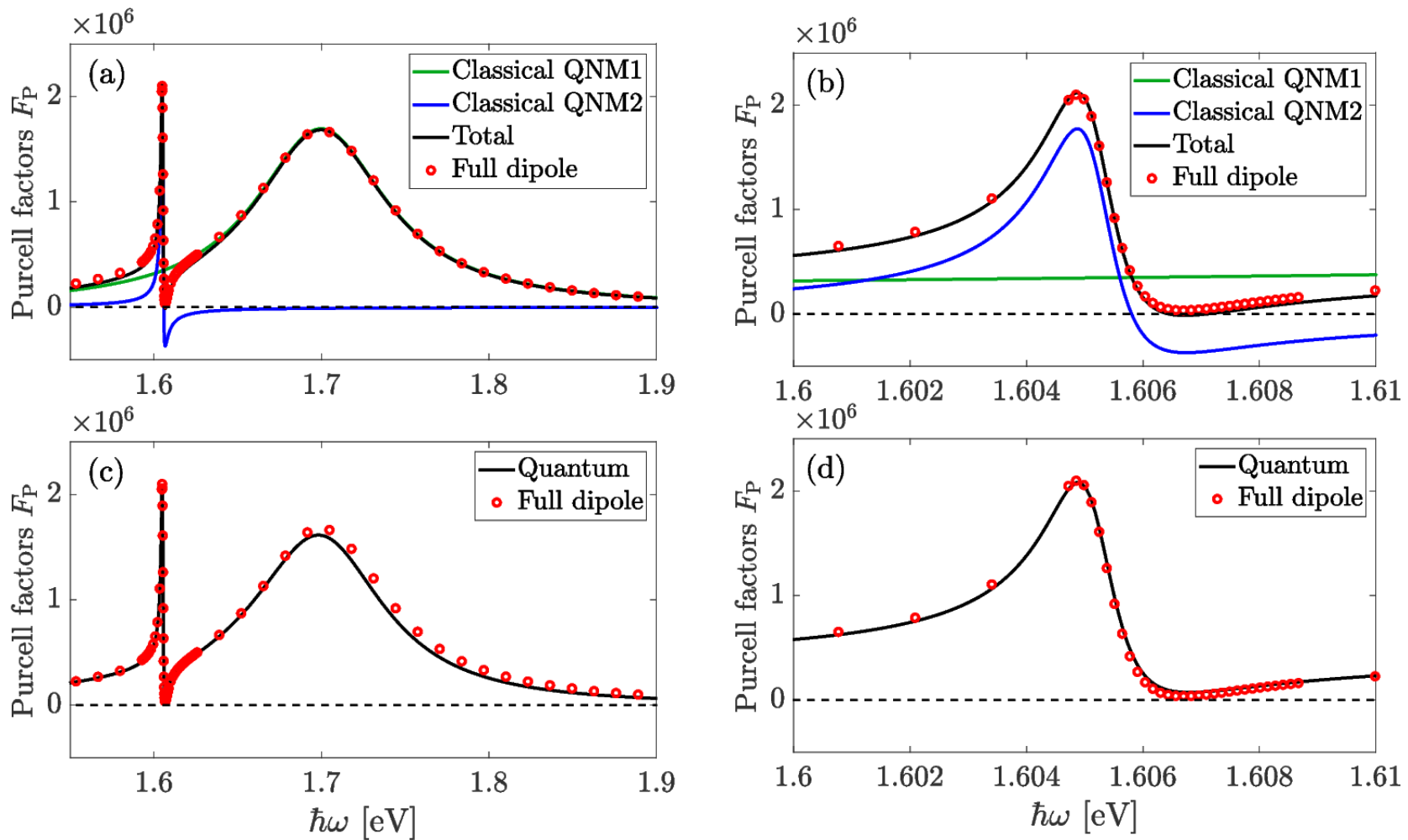

Figure 15. Purcell factors for a $z$-polarized point dipole at the dimer gap center point (as shown Fig .13) from (a) classical QNMs theory and (c) quantum theory. Panels (b) and (d) show a zoom in of (a) and (d) near the Fano resonance. The green curve is from QNMs theory (Eq. (16)), which show excellent qualitative agreement with full dipole calculation (red circles). There are two dominated modes with $\tilde{\omega}_{1}=1.6999-0.0479 i \mathrm{eV}, Q_{1}=17.8$ and $\tilde{\omega}_{1}=1.6052-0.0007 i \mathrm{eV}, Q_{2}=1139.3$. Black line and blue line present their respective contribution to Purcell factors. (c) Quantum Purcell factors (Eq. (47)) for coupled structures, which show nice agreement with full dipole results (red circles). The corresponding $4 S$ parameter are as follows, $S_{11}=0.894+0.068, S_{22}=0.904+0.134, S_{12}=(-0.0042-0.0967 i)+(-0.0021-0.0024 i), S_{21}=S_{12}^{*}$.

Table X. Pole $S_{\mathrm{p} 2,22}^{\mathrm{rad}}$ from Eq. (67) with $h=50 \mathrm{~nm}$ (surrounding entire coupled structures), quoted to the third decimal place. For the calculations, the PC-like mode uses a cuboid surface surrounding the entire structure.

\begin{tabular}{|c|c|c|}
\hline near field surface $h=50 \mathrm{~nm}$ & & \\
\hline angle grid for $\vartheta$ and $\varphi$ & run time & pole $S_{\mathrm{p} 2,22}^{\text {rad }}$ \\
\hline$\pi / 2$ & 4.6 mins & 0.194 \\
\hline$\pi / 5$ & $16.8 \mathrm{mins}$ & 0.150 \\
\hline$\pi / 10$ & $43.3 \mathrm{mins}$ & 0.140 \\
\hline$\pi / 15$ & $56.3 \mathrm{mins}$ & 0.137 \\
\hline$\pi / 20$ & 1.26 hours & 0.134 \\
\hline$\pi / 30$ & 1.7 hours & 0.134 \\
\hline
\end{tabular}

(black curve), which show nice agreement with full dipole results (red circles, Eq. (79)). Interestingly, the quantum result is net positive, since the quantum basis states constitute a different representation of the modes that are needed from quantum mechanical (namely, the symmetrized QNMs).

Given the extreme localization of a 2-nm gap, and the complex hybrid structure, the agreement with full dipole calculations is rather striking and shows the power of the
Table XI. Pole results for $S_{\mathrm{p} 2,12}^{\mathrm{rad}}$ from Eq. (67), quoted to the 4 th decimal place. For the calculations, the PC-like mode uses a cuboid surface surrounding the entire structure, while the plasmon-like mode uses a cuboid surface that surrounds the dimer.

\begin{tabular}{|c|c|c|}
\hline near field surface $h=50 \mathrm{~nm}$ & & \\
\hline angle grid for $\vartheta$ and $\varphi$ & run time & pole $S_{\mathrm{p} 2,12}^{\mathrm{rad}}$ \\
\hline$\pi / 2$ & $4.5 \mathrm{mins}$ & $-0.0062+0.0006 i$ \\
\hline$\pi / 3$ & $7.0 \mathrm{mins}$ & $-0.0040-0.0051 i$ \\
\hline$\pi / 5$ & $13.3 \mathrm{mins}$ & $-0.0023+0.0003 i$ \\
\hline$\pi / 10$ & $41.9 \mathrm{mins}$ & $-0.0019-0.0013 i$ \\
\hline$\pi / 20$ & $53.8 \mathrm{mins}$ & $-0.0021-0.0024 i$ \\
\hline$\pi / 30$ & 1.4 hours & $-0.0021-0.0024 i$ \\
\hline
\end{tabular}

quantum model, which can then be used to explore a wide range of nonlinear quantum optical effects.

\section{CONCLUSIONS}

In summary, we have introduced a timely and efficient NF2FF approach for QNMs, which allows one to quickly 
and accurately obtain the regularized QNMs in the far field. We also showed how to compliment this NF2FF transformation with an efficient pole approximation and a very far field extrapolation for obtaining the quantum optical parameters for quantized QNM theory ${ }^{33}$ : For example, the quantized QNM theories require the regularized fields flowing radiatively through a closed surface. In this regard, the NF2FF approach is shown to be several orders of magnitude faster to that of a direct Dyson equation approach, which has been used in the literature to obtain the regularized fields. In a practical calculation of a 3D metal dimer QNM, we obtain the desired quantization parameters in under 1 minute run time on a standard computer workstation, which previously takes weeks to one month of computational time. We also reported detailed numerical convergence checks on the QNM quantization parameters, both for the radiative and nonradiative contributions. The former requires the QNM field within or close to the metal (lossy structure), while the latter required the regularized QNM field (nondivergent), far outside the resonator. Together these contributions form the input-output channel contributions in quantum optics and form the basis for Fock space quantization for these dissipative modes. More generally, the regularized QNMs are the physical fields that connect to experiments for detection of the modes outside the resonator.

The general formalism presented here solves a major computational problem in quantized open-cavity mode theories by efficiently returning regularized QNMs for a wide range of structures, yielding QNM parameters for immediate use in quantum optics and quantum plasmonics with the underlying quantized open-cavity modes. We applied the theory to compute far-field (regularized) mode profiles and quantum optical parameters for quantized QNMs theory, for both metal dimer structures and a complex hybrid structure that formed coupled QNMs between a PC cavity and a dimer. In all cases, we show excellent agreement with full dipole classical calculations in the bad cavity limit, using both semiclassical and fully quantum simulations. This approach can thus easily be used as input to explore system level quantum optics with these modes, where unique few quanta effects beyond the single Fock state and coherent state can be probed and explored beyond the semiclassical limit. Such effects will be reported elsewhere.

\section{ACKNOWLEDGMENTS}

We acknowledge Queen's University and the Natural Sciences and Engineering Research Council of Canada for financial support, and CMC Microsystems for the provision of COMSOL Multiphysics to facilitate this research. We also acknowledge support from the Deutsche Forschungsgemeinschaft (DFG) through SFB 951 Project B12 (Project number 182087777), Project BR1528/82 (Project number 177864488) and the Alexander von Humboldt Foundation through a Humboldt Research
Award. We thank Mohsen Kamandar Dezfouli for useful discussions. This project has also received funding from the European Unions Horizon 2020 research and innovation program under Grant Agreement No. 734690 (SONAR).

Appendix A: Simpler calculation for $S_{\mathrm{p} 2,22}^{\mathrm{rad}}$ and $S_{\mathrm{p} 2,12}^{\mathrm{rad}}$, using only fields surrounding the dimer

In the main text, for the PC-like mode of the hybrid device we uses a cuboid surrounding the entire coupled structures to calculate the corresponding $S_{\mathrm{p} 2,22}^{\mathrm{rad}}$ and $S_{\mathrm{p} 2,22}^{\mathrm{rad}}$ (Eq. (67)). Here we show it is also a good approximation if the near field surface is replaced by a cuboid just surrounding the dimer. The grid sizes at this near field surface are set as $0.5 \mathrm{~nm}$ (the same as that used in main text). The convergence check over angle integral are shown in Table XII and XIII. We got $S_{\mathrm{p} 2,22}^{\mathrm{rad}}=0.061$, $S_{\mathrm{p} 2,12}^{\mathrm{rad}}=-0.0008-0.0063 i$, and $S_{\mathrm{p} 2,21}^{\mathrm{rad}}=S_{\mathrm{p} 2,12}^{\mathrm{rad} *}$. As also shown in these tables, the run times are only several seconds.

Table XII. Pole $S_{\mathrm{p} 2,22}^{\mathrm{rad}}$ from Eq. (67) with $h=50 \mathrm{~nm}$ (with small cuboid around the dimer), quoted to the third decimal place.

\begin{tabular}{|c|c|c|}
\hline near field surface $h=50 \mathrm{~nm}$ & & \\
\hline angle grid for $\vartheta$ and $\varphi$ & run time & pole $S_{\mathrm{p} 2,22}^{\text {rad }}$ \\
\hline$\pi / 2$ & 1.5 secs & 0.073 \\
\hline$\pi / 3$ & 2.8 secs & 0.062 \\
\hline$\pi / 5$ & 4.9 secs & 0.061 \\
\hline$\pi / 8$ & 9.4 secs & 0.061 \\
\hline$\pi / 10$ & 13.4 secs & 0.061 \\
\hline
\end{tabular}

A summary of the quantum parameters are now as follows: we use the previous $S_{\mathrm{p} 2,11}^{\mathrm{rad}}=0.068$ and just replace $S_{\mathrm{p} 2,22}^{\mathrm{rad}}$ and $S_{\mathrm{p} 2,12}^{\mathrm{rad}}$ with the new ones, $S_{\mathrm{p}, 11}=0.894+0.068$, $S_{\mathrm{p}, 22}=0.904+0.061, S_{\mathrm{p}, 12}=(-0.0042-0.0967 i)+$ $(-0.0008-0.0063 i), S_{\mathrm{p}, 21}=S_{\mathrm{p}, 12}^{*}$, where the first is nonradiative part and the second is radiative part. Then quantum Purcell factors (Eq. (47)) for the coupled structure is shown in Fig. 16, which show good agreement with full dipole results (red circles, Eq. (79)).

Table XIII. Pole $S_{\mathrm{p} 2,12}^{\mathrm{rad}}$ from Eq. (67) (both with small cuboid surface), quoted to the 4 th decimal place.

\begin{tabular}{|c|c|c|}
\hline near field surface $h=50 \mathrm{~nm}$ & & \\
\hline angle grid for $\vartheta$ and $\varphi$ & run time & pole $S_{\mathrm{p} 2,12}^{\mathrm{rad}}$ \\
\hline$\pi / 2$ & 4.0 secs & $-0.0010-0.0075 i$ \\
\hline$\pi / 3$ & 5.6 secs & $-0.0008-0.0064 i$ \\
\hline$\pi / 5$ & 10.2 secs & $-0.0008-0.0063 i$ \\
\hline$\pi / 8$ & 19.1 secs & $-0.0008-0.0063 i$ \\
\hline
\end{tabular}



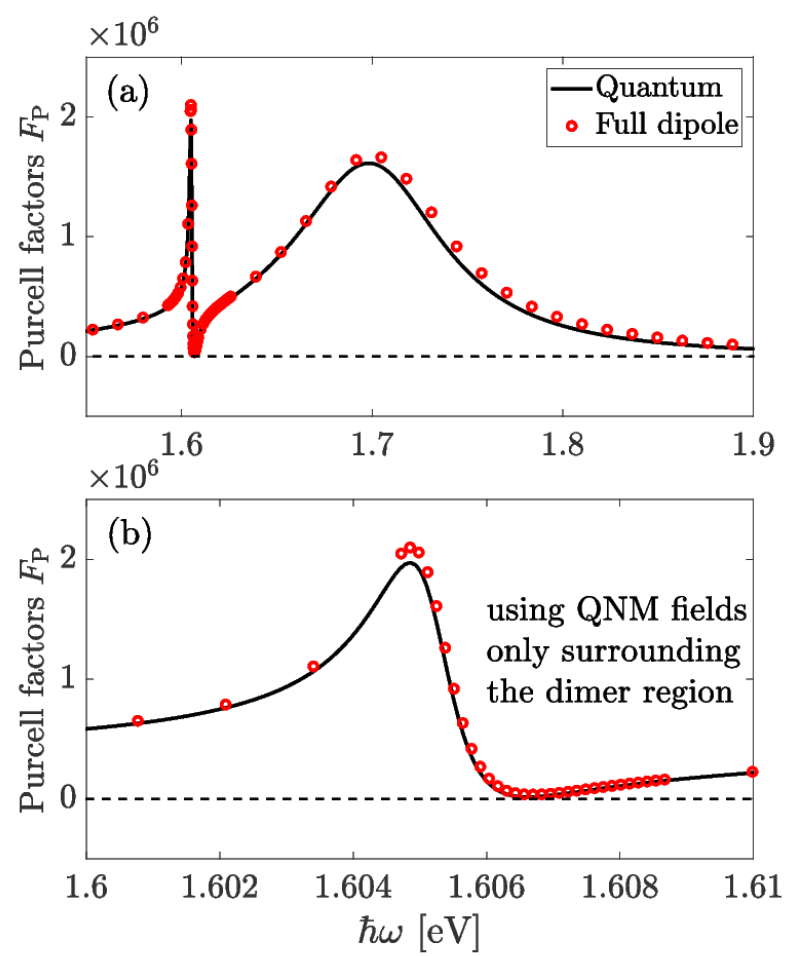

Figure 16. Quantum Purcell factors (Eq. (47)) for coupled structures, which again show good agreement with full dipole results (red circles). In contrast to the results shown in Figs. 15(c)-(d), when calculating $S_{\mathrm{p} 2, \mu \eta}^{\mathrm{rad}}$ (Eq. (67)), the near field surfaces are now chosen as a small cuboid $(h=50$ $\mathrm{nm})$ just surrounding dimer for both plasmon-like mode and PC-like mode. The corresponding $4 S$ parameter are as follows, $S_{11}=0.894+0.068, S_{22}=0.904+0.061, S_{12}=$ $(-0.0042-0.0967 i)+(-0.0008-0.0063 i), S_{21}=S_{12}^{*}$. In this way the run times to obtain the radiative $S$ parameters are reduced from a few hours to a few seconds, while still maintaining a very good level of accuracy with no fitting parameters.

*jr180@queensu.ca

1 K. J. Vahala, "Optical microcavities," Nature 424, 839-846 (2013).

2 Richard Kounai Chang and Anthony J Campillo, Optical processes in microcavities, Vol. 3 (World scientific, 1996).

3 D. J. Bergman and M. I. Stockman, "Surface plasmon amplification by stimulated emission of radiation: Quantum generation of coherent surface plasmons in nanosystems," Phys. Rev. Lett. 90, 027402 (2003).

4 Stefan Alexander Maier, Plasmonics: fundamentals and applications (Springer Science \& Business Media, 2007).

${ }^{5}$ M. A. Noginov, G. Zhu, A. M. Belgrave, R. Bakker, V. M. Shalaev, E. E. Narimanov, S. Stout, E. Herz, T. Suteewong, and U. Wiesner, "Demonstration of a spaser-based nanolaser," Nature 460, 1110-1112 (2009).

6 Lukas Novotny and Niek van Hulst, "Antennas for light," Nature Photonics 5, 83-90 (2011).

7 Darrick E. Chang, Anders S. Sørensen, Eugene A. Demler, and Mikhail D. Lukin, "A single-photon transistor using nanoscale surface plasmons," Nature Physics 3, 807 (2007).

8 Mads Lykke Andersen, Søren Stobbe, Anders Søndberg Sørensen, and Peter Lodahl, "Strongly modified plasmon-matter interaction with mesoscopic quantum emitters," Nature Physics 7, 215-218 (2011).

9 Zubin Jacob and Vladimir M. Shalaev, "Plasmonics goes quantum," Science 334, 463-464 (2011).

10 M. S. Tame, K. R. McEnery, Ş. K. Özdemir, J. Lee, S. A. Maier, and M. S. Kim, "Quantum plasmonics," Nature Physics 9, 329-340 (2013).

11 Pierre Berini and Israel De Leon, "Surface plasmon-polariton amplifiers and lasers," Nature Photonics 6, 16-24 (2012).

12 Rohit Chikkaraddy, Bart de Nijs, Felix Benz, Steven J. Barrow, Oren A. Scherman, Edina Rosta, Angela Demetriadou, Peter Fox, Ortwin Hess, and Jeremy J. Baumberg, "Single-molecule strong coupling at room temperature in plasmonic nanocavities," Nature 535, 127-130 (2016).

13 F. Benz, M. K. Schmidt, A. Dreismann, R. Chikkaraddy, 
Y. Zhang, A. Demetriadou, C. Carnegie, H. Ohadi, B. de Nijs, R. Esteban, J. Aizpurua, and J. J. Baumberg, "Single-molecule optomechanics in "picocavities"," Science 354, 726-729 (2016).

14 Philip M. Morse and Herman Feshbach, "Methods of theoretical physics," American Journal of Physics 22, 410-413 (1954).

15 P. T. Kristensen, C. Van Vlack, and S. Hughes, "Generalized effective mode volume for leaky optical cavities," Optics Letters 37, 1649 (2012).

16 Philip Trøst Kristensen and Stephen Hughes, "Modes and Mode Volumes of Leaky Optical Cavities and Plasmonic Nanoresonators," ACS Photonics 1, 2-10 (2014).

17 H. M. Lai, P. T. Leung, K. Young, P. W. Barber, and S. C. Hill, "Time-independent perturbation for leaking electromagnetic modes in open systems with application to resonances in microdroplets," Phys. Rev. A 41, 5187-5198 (1990).

18 P. T. Leung, S. Y. Liu, and K. Young, "Completeness and orthogonality of quasinormal modes in leaky optical cavities," Physical Review A 49, 3057-3067 (1994).

19 P. T. Leung, S. Y. Liu, S. S. Tong, and K. Young, "Timeindependent perturbation theory for quasinormal modes in leaky optical cavities," Physical Review A 49, 3068-3073 (1994).

20 P. T. Leung and K. M. Pang, "Completeness and timeindependent perturbation of morphology-dependent resonances in dielectric spheres," JOSAB 13, 805-817 (1996).

${ }^{21}$ KM Lee, PT Leung, and KM Pang, "Dyadic formulation of morphology-dependent resonances. i. completeness relation," JOSAB 16, 1409-1417 (1999).

${ }^{22}$ C. Sauvan, J. P. Hugonin, I. S. Maksymov, and P. Lalanne, "Theory of the Spontaneous Optical Emission of Nanosize Photonic and Plasmon Resonators," Physical Review Letters 110, 237401 (2013).

23 Q. Bai, M. Perrin, C. Sauvan, J.-P. Hugonin, and P. Lalanne, "Efficient and intuitive method for the analysis of light scattering by a resonant nanostructure," Optics Express 21, 27371-27382 (2013).

${ }^{24}$ Lin Zschiedrich, Felix Binkowski, Niko Nikolay, Oliver Benson, Günter Kewes, and Sven Burger, "Riesz-projectionbased theory of light-matter interaction in dispersive nanoresonators," Phys. Rev. A 98, 043806 (2018).

25 Philippe Lalanne, Wei Yan, Kevin Vynck, Christophe Sauvan, and Jean-Paul Hugonin, "Light interaction with photonic and plasmonic resonances," Laser \& Photonics Reviews 12, 1700113 (2018).

${ }^{26}$ Philip Trøst Kristensen, Kathrin Herrmann, Francesco Intravaia, and Kurt Busch, "Modeling electromagnetic resonators using quasinormal modes," (2019), arXiv:1910.05412.

27 E. A. Muljarov, W. Langbein, and R. Zimmermann, "Brillouin-wigner perturbation theory in open electromagnetic systems," EPL 92, 50010 (2010).

${ }^{28}$ Rong-Chun Ge, Philip Trøst Kristensen, Jeff F Young, and Stephen Hughes, "Quasinormal mode approach to modelling light-emission and propagation in nanoplasmonics," New Journal of Physics 16, 113048 (2014).

29 Mohsen Kamandar Dezfouli, Reuven Gordon, and Stephen Hughes, "Modal theory of modified spontaneous emission of a quantum emitter in a hybrid plasmonic photonic-crystal cavity system," Phys. Rev. A 95, 013846 (2017).

30 Antonio I. Fernández-Domínguez, Sergey I. Bozhevolnyi, and N. Asger Mortensen, "Plasmon-enhanced generation of nonclassical light," ACS Photonics 5, 3447-3451 (2018).

31 K. C. Ho, P. T. Leung, Alec Maassen van den Brink, and K. Young, "Second quantization of open systems using quasinormal modes," Phys. Rev. E 58, 2965-2978 (1998).

32 S. Severini, A. Settimi, C. Sibilia, M. Bertolotti, A. Napoli, and A. Messina, "Second quantization and atomic spontaneous emission inside one-dimensional photonic crystals via a quasinormal-modes approach," Phys. Rev. E 70, 056614 (2004).

33 Sebastian Franke, Stephen Hughes, Mohsen Kamandar Dezfouli, Philip Trøst Kristensen, Kurt Busch, Andreas Knorr, and Marten Richter, "Quantization of quasinormal modes for open cavities and plasmonic cavity quantum electrodynamics," Phys. Rev. Lett. 122, 213901 (2019).

34 Paul A Martin, Multiple scattering: interaction of timeharmonic waves with $N$ obstacles, 107 (Cambridge University Press, 2006).

35 Mohsen Kamandar Dezfouli and Stephen Hughes, "Regularized quasinormal modes for plasmonic resonators and open cavities," Physical Review B 97, 115302 (2018).

36 M.J. Barth, R.R. McLeod, and R.W. Ziolkowski, "A near and far-field projection algorithm for finite-difference timedomain codes," Journal of Electromagnetic Waves and Applications 6, 5-18 (1992).

37 John B Schneider, Understanding the Finite-Difference Time-Domain Method, www. eecs.wsu. edu/ ₹chneidj/ ufdtd, 2010.

38 Stephen Hughes, Sebastian Franke, Chris Gustin, Mohsen Kamandar Dezfouli, Andreas Knorr, and Marten Richter, "Theory and limits of on-demand single-photon sources using plasmonic resonators: A quantized quasinormal mode approach," ACS Photonics 6, 2168-2180 (2019).

39 Philip Trøst Kristensen, Rong-Chun Ge, and Stephen Hughes, "Normalization of quasinormal modes in leaky optical cavities and plasmonic resonators," Phys. Rev. A 92, 053810 (2015).

40 Philip Trost Kristensen, Jakob Rosenkrantz de Lasson, Mikkel Heuck, Niels Gregersen, and Jesper Mork, "On the theory of coupled modes in optical cavity-waveguide structures," Journal of Lightwave Technology 35, 42474259 (2017).

41 Pascal Anger, Palash Bharadwaj, and Lukas Novotny, "Enhancement and quenching of single-molecule fluorescence," Phys. Rev. Lett. 96, 113002 (2006).

42 Rémi Colom, Ross McPhedran, Brian Stout, and Nicolas Bonod, "Modal expansion of the scattered field: Causality, nondivergence, and nonresonant contribution," Phys. Rev. B 98, 085418 (2018).

43 S. A. Schelkunoff, "ome equivalence theorems of electromagnetics and their application to radiation problems," Bell Labs Technical Journal 15, 92 (1936).

44 Ho Trung Dung, Ludwig Knöll, and Dirk-Gunnar Welsch, "Three-dimensional quantization of the electromagnetic field in dispersive and absorbing inhomogeneous dielectrics," Phys. Rev. A 57, 3931-3942 (1998).

45 L. G. Suttorp and A. J. van Wonderen, "Fano diagonalization of a polariton model for an inhomogeneous absorptive dielectric," EPL 67, 766 (2004).

46 J. I. Cirac, "Interaction of a two-level atom with a cavity mode in the bad-cavity limit," Phys. Rev. A 46, 4354-4362 (1992).

47 COMSOL Inc., "Comsol multiphysics v 5.4," www. comsol. com 
48 Keita Sasada, Naomichi Hatano, and Gonzalo Ordonez, "Resonant spectrum analysis of the conductance of an open quantum system and three types of fano parameter," Journal of the Physical Society of Japan 80, 104707 (2011).

49 Niket Thakkar, Morgan T. Rea, Kevin C. Smith, Kevin D. Heylman, Steven C. Quillin, Kassandra A. Knapper, Erik H. Horak, David J. Masiello, and Randall H. Goldsmith, "Sculpting fano resonances to control photonic-plasmonic hybridization," Nano Letters 17, 69276934 (2017).

50 Michael Barth, Stefan Schietinger, Sabine Fischer, Jan Becker, Nils Nüsse, Thomas Aichele, Bernd Löchel, Carsten Sönnichsen, and Oliver Benson, "Nanoassembled plasmonic-photonic hybrid cavity for tailored light-matter coupling," Nano Letters 10, 891-895 (2010).

51 Hugo M. Doeleman, Ewold Verhagen, and A. Femius Koenderink, "Antenna-cavity hybrids: Matching polar opposites for purcell enhancements at any linewidth," ACS Photonics 3, 1943-1951 (2016).

52 Isabelle M. Palstra, Hugo M. Doeleman, and A. Femius Koenderink, "Hybrid cavity-antenna systems for quantum optics outside the cryostat?" Nanophotonics 8, 1513-1531 (2019).

53 Mohsen Kamandar Dezfouli, Reuven Gordon, and Stephen Hughes, "Molecular optomechanics in the anharmonic cavity-QED regime using hybrid metal-dielectric cavity modes," ACS PHOTONICS 6, 1400-1408 (2019). 